\title{
A PARAMETRIX FOR KOHN'S OPERATOR
}

\author{
BRIAN STREET
}

\begin{abstract}
Recently, Kohn constructed examples of sums of squares of complex vector fields satisfying Hörmander's condition that lose derivatives, but are nevertheless hypoelliptic. He also demonstrated optimal $L^{2}$ regularity. In this paper, we construct parametricies for Kohn's operators, which lead to the corresponding $L^{p}(1<p<\infty)$ and Lipschitz regularity. In fact, our parametrix construction generalizes to a somewhat larger class of operators, yielding some new examples of operators which are hypoelliptic, but lose derivatives.
\end{abstract}

\section{INTRODUCTION}

Suppose

$$
X_{0}, X_{1}, \ldots, X_{n}
$$

are real vector fields. In a seminal paper, [Hör67], Hörmander proved that an operator of the form

$$
\mathcal{L}=\sum_{j=1}^{n} X_{j}^{2}+X_{0}
$$

is hypoelliptic provided

$$
X_{0}, \ldots, X_{n},\left[X_{i}, X_{j}\right],\left[\left[X_{i}, X_{j}\right], X_{k}\right], \ldots
$$

span the tangent space at every point. That is, if the $X_{i}$ s along with commutators of all orders span the tangent space at every point. By hypoelliptic, we mean that if $u$ is a distribution and $\mathcal{L} u$ is $C^{\infty}$ near a point $\xi$, then $u$ is $C^{\infty}$ near $\xi$. In fact, he proved that $\mathcal{L}$ is subelliptic, that is, there exists an $\epsilon>0$ (depending on $\xi$ ) such that if $\mathcal{L} u \in L_{s}^{2}$ near $\xi$, then $u \in L_{s+\epsilon}^{2}$ near $\xi$ (here, $L_{s}^{2}$ is the standard $L^{2}$ Sobolev space). The $\epsilon$, above, only depends on the number of commutators it takes to span the tangent space near $\xi$.

Following Hörmander's result, Rothschild and Stein [RS76] constructed parametricies for the above operators of Hörmander (and for more general operators). These parametrices yield proofs of the optimal regularity for $\mathcal{L}$ in Lipschitz and $L^{p}$ Sobolev spaces $(1<p<\infty)$.

Recently, Kohn [Koh05] studied the analogous question with complex vector fields in place of real vector fields. He showed that the subellipticity does not hold for general complex vector fields. More specifically, he showed:

Theorem 1.1. For $k>0$ an integer, there exist complex vector fields $X_{1, k}$ and $X_{2}$ on a neighborhood of the $0 \in \mathbb{R}^{3}$ such that $X_{1, k}, X_{2}$, and their commutators of order $k+1$ span the tangent space near 0 , and such that if we set $\mathcal{A}=-X_{1, k}^{*} X_{1, k}-$ $X_{2}^{*} X_{2}$, then $\mathcal{A}$ is not subelliptic near 0 , however it is hypoelliptic near 0 . Here, * 
denotes the $L^{2}$ adjoint. Moreover, $\mathcal{A}$ "loses precisely $k-1$ derivatives near 0 in $L^{2}$ Sobolev spaces;" that is, there exists a neighborhood $U$ of 0 such that if $u$ is a distribution, $\xi \in U$, and $\mathcal{A} u$ is in $L_{s}^{2}$ on a neighborhood of $\xi$, then $u$ is in $L_{s-k+1}^{2}$ on a neighborhood of $\xi$-and one can say no better in general. See Section 1.4 for the definition we will use of loss of derivatives.

In the appendix to [Koh05], Derridj and Tartakoff prove that Kohn's operator is analytic hypoelliptic. Soon after Kohn's paper appeared, Bove, Derridj, Kohn, and Tartakoff [BDKT06] used the same methods to prove hypoellipticity, analytic hypoellipticity, and optimal $L^{2}$ Sobolev regularity for a larger class of operators. At around the same time as Kohn's paper appeared, Parenti and Parmeggiani [PP05] constructed parametrices for a certain class of pseudodifferential operators that were hypoelliptic but lost derivatives. Subsequently, they showed ([PP06]) that their results could be applied to operators related to Kohn's example, yielding a parametrix construction and a proof of hypoellipticity. Their methods use the pseudodifferential calculus of [BdM74] (and also involve conjugation by a Fourier integral operator), and so do not seem to yield any sort of $L^{p}$ or Lipschitz regularity (we discuss the relationship between their results and ours in Section 5). Christ [Chr05] showed that a sum of squares of complex vector fields whose commutators span the tangent space at every point may not be hypoelliptic at all. In a paper which is seemingly unrelated to the above papers, Journé and Trépreau [JT06] study systems of complex vector fields that are hypoelliptic but lose derivatives. They prove regularity results in $L^{2}$ Sobolev spaces.

Prior to the above results, Heller (in his thesis, [Hel86]) expanded on an idea of Stein ([Ste82]) and proved $L^{p}$ regularity (and analytic hypoellipticity) by a parametrix construction for certain left invariant differential operators on the Heisenberg group, that lose derivatives. His results do not apply to Kohn's operator, since Kohn's operator is not left invariant.

Some of the results of this paper were announced in [Str06], though the methods involved here are more complicated than those that were discussed in that paper. Moreover, we present here methods which prove Lipschitz regularity, give a more precise form of the parametrix, and yield better understanding of what terms are "lower order."

\section{Acknowledgements}

This paper represents my doctoral dissertation at Princeton University. I would like to thank my advisor, Professor Elias Stein, for his constant support and encouragement during this project, and for all of his valuable suggestions and the great mathematics he taught me along the way. I would also like to thank Professor Joseph Kohn for some very interesting conversations about this project.

1.1. Statement of Results. The purpose of this paper is to construct a parametrix for Kohn's operator, which proves optimal regularity in $L^{p}$ Sobolev spaces and Lipschitz spaces. In fact, we will be able to construct our parametrix for a somewhat more general class of operators (see below), which are not necessarily a sum of squares (indeed, not even necessarily positive operators on $L^{2}$ ). This parametrix demonstrates what sort of terms can be considered "lower order", as well as proves Lipschitz and $L^{p}$ Sobolev regularity (which we will show to be optimal for a subclass of our operators). In the particular case of Kohn's operator, 
the parametrix shows that $\mathcal{A}$ loses precisely $k-1$ derivatives in $L^{p}$ Sobolev spaces $(1<p<\infty)$, and precisely $k-1$ derivatives in Lipschitz spaces.

Let $Z_{L}=\partial_{z}+i \bar{z} \partial_{t}$ and $\bar{Z}_{L}=\partial_{\bar{z}}-i z \partial_{t}$ (as operators on $\mathbb{R}^{3}$; these are actually left invariant vector fields on the Heisenberg group-see Section 2). Fix integers $a$ and $b$ such that $0 \leq b \leq a$ and $0<a$ and fix $t_{0} \in \mathbb{R}$. Define the operators:

$$
\begin{gathered}
A_{1}=Z_{L} \bar{Z}_{L} \\
A_{2}=\bar{Z}_{L}\left(z^{a} \bar{z}^{b} h(z, t)\right) Z_{L} \\
\mathcal{A}=A_{1}+A_{2}+\mathcal{A}_{l}
\end{gathered}
$$

where $h \in C^{\infty}\left(\mathbb{H}^{1}\right), h\left(0, t_{0}\right) \neq 0$, and $\mathcal{A}_{l}$ will be a "lower order" term whose form will be made precise below. We will construct a left parametrix for $\mathcal{A}$ near $\left(0, t_{0}\right)$. This parametrix will imply:

Theorem 1.2. $\mathcal{A}$ is hypoelliptic on a neighborhood of $\left(0, t_{0}\right)$. Moreover, $\mathcal{A}$ loses at most $\frac{a+b}{2}-1$ derivatives near $\left(0, t_{0}\right)$ in $L^{p}$ Sobolev spaces $(1<p<\infty)$ and Lipschitz spaces. Under an added assumption on $\mathcal{A}_{l}$ (see Section 4) these results are optimal; that is, $\mathcal{A}$ loses precisely $\frac{a+b}{2}-1$ derivatives near $\left(0, t_{0}\right)$ in $L^{p}$ Sobolev spaces and Lipschitz spaces. See Section 1.4 for the definitions of loss that we are using.

In fact, our parametrix will prove sharper regularity results, which we will make precise later on (see Section 3.3 and Section 3.4). The proof of optimality can be found in Section 4. It is worth noting that when $a=b, h=1$, and $\mathcal{A}_{l}=0$, this result implies Kohn's result-see Section 1.3.

To define $\mathcal{A}_{l}$, define the operators:

$$
\begin{gathered}
A_{3}=Z_{L} g^{(1)}(z, t) \\
A_{4}=\left(\sum_{\substack{d+e=a+b \\
0 \leq d, e}} z^{d} \bar{z}^{e} g_{d, e}^{(2)}(z, t)\right) \bar{Z}_{L} \\
A_{5}=\sum_{\substack{d+e=a+b-1 \\
0 \leq d, e}} z^{d} \bar{z}^{e} g_{d, e}^{(3)}(z, t) \\
A_{6}=\bar{Z}_{L}\left(\begin{array}{c}
\sum_{\substack{d+e=a+b+1 \\
0 \leq d, e}} z^{d} \bar{z}^{e} g_{d, e}^{(4)}(z, t) \\
\mathcal{A}_{l}=A_{3}+A_{4}+A_{5}+A_{6}
\end{array}\right) Z_{L}
\end{gathered}
$$

where $g^{(1)}$ and the $g_{d, e}^{(i)}$ are arbitrary $C^{\infty}$ functions.

The terms $A_{3}, A_{4}, A_{5}$, and $A_{6}$ should be each considered as "lower order." Indeed, if $J$ is the parametrix we will construct for $A_{1}+A_{2}$, we will see that $J A_{3}$, $J A_{4}$, and $J A_{5}$ are all smoothing of order $1^{-}$(see Section 2.4 for definitions of orders of smoothing), and therefore these terms are genuinely lower order. $J A_{6}$ will be smoothing of order $0^{-}$but will vanish as $z \rightarrow 0$, and so will be lower order in a much more delicate sense, which we describe in Section 3.4. 
Remark 1.3. These terms are lower order with respect to a left parametrix. If we were considering a right parametrix, we would have to take the adjoint of each of the above terms. For instance, the reader may note that $A_{3}^{*}=0$ on the kernel of $A_{1}$, which would come into play in the construction of the right parametrix. In this paper, we will be using the adjoint of this fact.

Our parametrix construction happens entirely at the operator level. We do not take any Fourier transforms, or microlocalize in any way. All of our localizations will be trivial, as the operators we are using are pseudolocal. This allows us to avoid most of the difficulties of Kohn's proof (see Section 1.3). Perhaps the greatest difficulty that arises in our proof is the explicit inversion of a certain Toeplitz operator (see Section 3.2); and generalizing our method seems dependent on finding a better way of dealing with such operators.

1.2. Basic Notation. We offer the reader a quick introduction to our notation. $\mathbb{H}^{1}$ will denote the 3 dimensional Heisenberg group (see Section 2). For coordinates in $\mathbb{H}^{1}$, we will use $(z, t) \in \mathbb{H}^{1}$, with $z \in \mathbb{C}$ and $t \in \mathbb{R}$. $z$ and $\bar{z}$ will be complex numbers, while $t$ will be a real number, and $\xi, \eta$, and $\zeta$ will be elements of $\mathbb{H}^{1}$. We will write $L_{s}^{p}(s \in \mathbb{R})$ for the standard $L^{p}$ Sobolev spaces, and $\Lambda_{\alpha}(\alpha>0)$ for the standard Lipschitz spaces (for a background on Lipschitz spaces, see [Ste70]). $L^{(j)}$ will stand for a homogeneous (of order $j$ ) left invariant convolution operator, whose convolution kernel is smooth away from 0 (see Section 2.1). Similarly, $R^{(j)}$ will stand for a homogeneous right invariant convolution operator. Usually, a number in a superscript (like $E^{(j)}$ ) will signify the degree of smoothing of the operator (as defined in Section 2.4). A problem arises, with (for instance) operators like $L^{(0)}$ which are smoothing of order $0^{-}$(also defined in Section 2.4). We will be explicit when this difference matters. Terms like $L^{(j)}$ or $R^{(j)}$ will stand for arbitrary elements of that form. Thus, for instance,

$$
L^{(0)} L^{(0)}
$$

really means a product of an arbitrary pair of such operators, not one operator squared.

For $\phi, \psi \in C_{0}^{\infty}, \phi \prec \psi$ means that $\psi$ is equal to 1 on a neighborhood of the support of $\phi$. When we write functions in our formulas, we will (almost always) mean them as multiplication operators. When we do not mean them as operators (and instead mean them as being in the domain of our operator) we will usually use $u$ or $v$ (which will usually mean an arbitrary distribution with compact support); in any case, it will be clear from context whether we mean to use the functions as multiplication operators or not.

For operators that do not necessarily commute, we will use ordered multi-index notation. For instance, if $A$ and $B$ do not commute, and $I$ is a sequence of 1 s and $2 \mathrm{~s}$, say $I=(1,1,2,1)$, then we have:

$$
(A, B)^{I}=A A B A
$$

and we write $|I|=4$, ie the length of the sequence. Also, if $A_{j}, j=1, \ldots, n$, is a sequence of noncommuting operators, we write:

$$
\prod_{j=1}^{n} A_{j}=A_{1} A_{2} \cdots A_{j}
$$


For two operators $A$ and $B$ (taking $C_{0}^{\infty}$ to distributions), we will use the notation $A=B$ to mean equality on $C_{0}^{\infty}$. This equality will extend (by continuity) to distributions with compact support in essentially every place we use it. If $A$ and $B$ are two operators taking distributions with compact support to distributions, we will write $A \equiv B$ if $A-B$ maps to $C^{\infty}$, ie the Schwartz kernel of $A-B$ is $C^{\infty}$.

We will have occasion to use holomorphic maps from an open set in $\mathbb{C}$ to a Fréchet space. We will say such a map, $f$, has a pole of order $j$ at $z_{0}$ if $\left(z-z_{0}\right)^{j} f$ extends to a holomorphic function on a neighborhood of $z_{0}$ (and does so for no lesser $j$ ). We will call a map whose set of poles is discrete, a meromorphic function. All of the poles in the functions we consider will be simple (of order 1), so there is no need to discuss essential singularities.

We will call a map, $f$, mapping an open subset of $\mathbb{R}^{n}$ to a Fréchet space, smooth if it is infinitely differentiable (in the sense of taking limits of difference quotients in the topology of the Fréchet space). Thus, holomorphic maps are smooth.

Finally, in our estimates, we will use the notation $\|u\|_{-\infty}$, where $u$ is a distribution of compact support. If we write:

$$
A \lesssim B+\|u\|_{-\infty}
$$

we mean, for every $N \in \mathbb{N}$, there exists a $C>0$ such that:

$$
A \leq C\left(B+\|u\|_{L_{-N}^{2}}\right)
$$

Since every distribution with compact support is in $L_{-N}^{2}$ for some $N$, this is the best sort of error term we can hope for.

1.3. Kohn's Proof. If we define $Z_{L}=\partial_{z}+i \bar{z} \partial_{t}$ and $\bar{Z}_{L}=\partial_{\bar{z}}-i z \partial_{t}$ (as in Section 1.1) then Kohn's operator takes the form:

$$
Z_{L} \bar{Z}_{L}+\bar{Z}_{L}|z|^{2 k} Z_{L}=-\left(\bar{Z}_{L}\right)^{*} \bar{Z}_{L}-\left(\bar{z}^{k} Z_{L}\right)^{*} \bar{z}^{k} Z_{L}
$$

(where $k>0$ is an integer).

Loss of derivatives complicates the usual proofs of hypoellipticity. Indeed, if one were to want to apply Hörmander's methods (see [Hör67]) to Kohn's operator, then one would start with an a priori estimate like:

$$
\|u\|_{L^{2}}+\left\|Z_{L} u\right\|_{L_{k-1}^{2}}+\left\|\bar{z}^{k} \bar{Z}_{L} u\right\|_{L_{k-1}^{2}} \lesssim\left|\langle\mathcal{A} u, u\rangle_{L_{k-1}^{2}}\right|+\|u\|_{-\infty}
$$

for all $u \in C^{\infty}$ with support in some small, but fixed, compact neighborhood around 0 (see [BDKT06], Proposition 1.1). Then one would like to localize this estimate. That is, we replace $u$ with $\phi u$, and try and commute $\phi$ past $\mathcal{A}$ with error terms that we can control. Unfortunately, that leaves error terms like:

$$
\left\|\phi^{\prime} u\right\|_{L_{k-1}^{2}}
$$

where $\phi \prec \phi^{\prime}$. This is much worse that what we were trying to bound. One thing Kohn realized was that $\left\|\phi^{\prime} u\right\|_{L_{k-1}^{2}}$ was far too coarse an estimate. In fact, if we imagine that $\phi$ was only localizing in the $z$ variable, we see that we need only estimate a distribution that vanishes as $z \rightarrow 0$ (since $[\mathcal{A}, \phi]$ is zero on a neighborhood of 0 in the $z$ variable). Kohn proved that, in this case, one can trade vanishing in $z$ at 0 for regularity. Our analog of this concept can be found in Proposition 3.4. 
A similar problem occurs when one wishes to raise the estimate (1.1) to higher Sobolev norms. Ie, if we wish to show an estimate like:

$$
\|u\|_{L_{s}^{2}} \lesssim\|\mathcal{A} u\|_{L_{s+k-1}^{2}}+\|u\|_{-\infty}
$$

Usually, one would replace $u$ in Equation (1.1) by something like

$$
(1-\triangle)^{s / 2} u
$$

Again, commuting the pseudodifferential operator $(1-\triangle)^{s / 2}$ past $\mathcal{A}$ leaves errors that are worse than what we were originally trying to bound. To take care of this problem, Kohn uses microlocalization techniques.

We will not be too detailed in the description of Kohn's argument, since it is quite complicated. We refer the reader to [BDKT06] and [Koh05] for details. However, we will briefly discuss Kohn's microlocalization techniques.

Let $\tau$ be dual to $t$ and $\sigma$ dual to $(x, y)$. Kohn creates three microlocalizations:

- The 0 microlocalization: $|\sigma| \gtrsim|\tau|$

- The + microlocalization: $\tau \gtrsim|\sigma|$

- The - microlocalization: $-\tau \gtrsim|\sigma|$

Kohn proves that on the 0 microlocalization, $\mathcal{A}$ is elliptic, and on the - microlocalization, $\mathcal{A}$ is subelliptic. Our analog of this result can be found in Theorem 3.3 (neither result implies the other directly, but they are similar). These results are analogous since the operator $\pi^{(L)}$ (the Cauchy-Szegö projection) is micro-supported on the + microlocalization. On the + microlocalization, the $L_{s}^{2}$ norm can be defined in terms of the pseudodifferential operator $\left(1+D_{t}\right)^{s}$ (which is a genuine pseudodifferential operator on the + microlocalization). $\left(1+D_{t}\right)^{s}$ commutes with $\mathcal{A}$, thereby removing the problem discussed above.

1.4. Loss Of Derivatives. There are many possible definitions of loss of derivatives. We take the following definition:

Definition 1.4. Let $\mathcal{A}$ be a differential operator on a manifold $M$, let $U \subset M$ be an open set, and fix $1<p<\infty$ and $s_{0} \in \mathbb{R}$. We say that $\mathcal{A}$ loses at most $r$ derivatives on $U$ in $L_{s_{0}}^{p}$ if for every $\phi \prec \phi^{\prime}$ with $\phi, \phi^{\prime} \in C_{0}^{\infty}(U)$ and for every distribution $u$ on $U$, we have:

$$
\|\phi u\|_{L_{s_{0}}^{p}} \lesssim\left\|\phi^{\prime} \mathcal{A} u\right\|_{L_{s_{0}+r}^{p}}+\left\|\phi^{\prime} u\right\|_{-\infty}
$$

(where the LHS being infinite implies the RHS is infinite, and the RHS being finite implies the LHS is finite). If $\mathcal{A}$ loses at most $r$ derivatives on $U$ in $L_{s_{0}}^{p}$, and does not lose at most $r-\epsilon$ derivatives on $U$ in $L_{s_{0}}^{p}$, for every $\epsilon>0$, we say that $\mathcal{A}$ loses precisely $r$ derivatives on $U$ in $L_{s_{0}}^{p}$. If $\mathcal{A}$ loses at most $r$ derivatives on $U$ in $L_{s_{0}}^{p}$ for every $s_{0}$, we say that $\mathcal{A}$ loses at most $r$ derivatives on $U$ in $L^{p}$ Sobolev spaces (here, and for the rest of the definition, $p$ remains fixed). If $\mathcal{A}$ loses precisely $r$ derivatives on $U$ in $L_{s_{0}}^{p}$ for every $s_{0}$, we say that $\mathcal{A}$ loses precisely $r$ derivatives on $U$ in $L^{p}$ Sobolev spaces.

For $\xi \in M$, we use "loses derivatives near $\xi$ " to denote the existence of an appropriate $U$. For instance " $\mathcal{A}$ loses precisely $r$ derivatives near $\xi$ in $L^{p}$ Sobolev spaces," means that there exists a neighborhood $U$ with $\xi \in U$, such that $\mathcal{A}$ loses precisely $r$ derivatives on $U$ in $L^{p}$ Sobolev spaces. 
For loss in Lipschitz spaces, merely replace $L_{s}^{p}$ with $\Lambda_{s}$ in the above (with the restriction that the parameter in $\Lambda_{s}$ must be $>0$, wherever it is used). Where we said " $L^{p}$ Sobolev spaces," we change to "Lipschitz spaces."

Remark 1.5. The above definition is not universally used. Some authors replace our $r$ with $r+m$, where $m$ is the degree of $\mathcal{A}$.

By the Sobolev imbedding theorem, if $U$ is a fixed open set and if for every $s$, there exists $r(s)$ such that $\mathcal{A}$ loses at most $r(s)$ derivatives on $U$ in $L_{s}^{p}$, then $\mathcal{A}$ is hypoelliptic on $U$. In particular, if $\mathcal{A}$ loses at most $r$ derivatives in $L^{p}$ Sobolev spaces, then $\mathcal{A}$ is hypoelliptic.

\section{The Heisenberg group}

Kohn's example takes place on the so-called three dimensional Heisenberg group, $\mathbb{H}^{1}$. $\mathbb{H}^{1}$ is a 3 -dimensional, step 2, nilpotent Lie group, which is diffeomorphic to $\mathbb{C} \times \mathbb{R}$ as a manifold. We will let $(z, t) \in \mathbb{C} \times \mathbb{R}$ denote coordinates on the Heisenberg group. In these coordinates, the multiplication is given by:

$$
\left(z_{1}, t_{1}\right)\left(z_{2}, t_{2}\right)=\left(z_{1}+z_{2}, t_{1}+t_{2}+2 \operatorname{Im}\left(z_{1} \overline{z_{2}}\right)\right)
$$

Thus, the identity of $\mathbb{H}^{1}$ is $0 \in \mathbb{C} \times \mathbb{R}$. If we write $z=x+i y$, then the Lie algebra of $\mathbb{H}^{1}$ is spanned by:

$$
X_{L}=\partial_{x}+2 y \partial_{t}, \quad Y_{L}=\partial_{y}-2 x \partial_{t}, \quad\left[X_{L}, Y_{L}\right]=-4 \partial_{t}
$$

here we have used the subscript $L$ to denote that these are left-invariant vector fields. We will later need both right and left invariant vector fields.

Defining $Z_{L}=\frac{1}{2}\left(X_{L}-i Y_{L}\right)$ and $\bar{Z}_{L}=\frac{1}{2}\left(X_{L}+i Y_{L}\right)$ (these are the same vector fields as in Section 1.1), we see that Kohn's operator is of the form:

$$
Z_{L} \bar{Z}_{L}+\bar{Z}_{L}|z|^{2 k} Z_{L}
$$

"Freezing coefficients" in the above, leaves the operator

$$
\mathcal{A}_{\beta}=Z_{L} \bar{Z}_{L}+\beta \bar{Z}_{L} Z_{L}
$$

where $\beta \in \mathbb{C}$. $\mathcal{A}_{\beta}$ is a left invariant differential operator on $\mathbb{H}^{1}$, and in this section we will review the relevant theories of such operators. For more background on $\mathbb{H}^{1}$ and its higher dimensional analogs, we refer the reader to [Ste93]. [Ste93] and [Fol75] together contain almost all the results that will be presented in this section, and we refer the reader to those two sources for more details and proofs.

$\mathbb{H}^{1}$ has a natural one parameter family of dilations, given by

$$
\gamma_{r}(z, t)=\left(r z, r^{2} t\right)
$$

here $0<r \in \mathbb{R}$ and $(z, t) \in \mathbb{H}^{1}$. It is easy to see that, for each $0<r \in \mathbb{R}, \gamma_{r}$ defines an automorphism of $\mathbb{H}^{1}$. Differentiating this map, for each $0<r \in \mathbb{R}$, we get an automorphism of the Lie algebra (which we will also denote by $\gamma_{r}$ ), given by

$$
\gamma_{r}\left(X_{L}\right)=r X_{L}, \quad \gamma_{r}\left(Y_{L}\right)=r Y_{L}, \quad \gamma_{r}\left(\left[X_{L}, Y_{L}\right]\right)=r^{2}\left[X_{L}, Y_{L}\right]
$$

For convenience, we will sometimes denote elements of $\mathbb{H}^{1}$ by $(z, t)=\zeta \in \mathbb{H}^{1}$. Euclidean measure on $\mathbb{C} \times \mathbb{R}$ is Haar measure on $\mathbb{H}^{1}$, and satisfies:

$$
d\left(\gamma_{r} \zeta\right)=r^{4} d \zeta
$$


we therefore call 4 the "homogeneous dimension" of $\mathbb{H}^{1}$. To accent its role in the theorems that follow, we will denote it by $Q$. Almost all of the results in this section hold for more general nilpotent Lie groups, and details may be found in [Fol75].

2.1. Left Invariant Convolution Operators. In this section, we will review the theory of convolution operators on $\mathbb{H}^{1}$. We will be concerned with convolution by certain "homogeneous distributions," and the theory of such operators was laid out in great generality by Folland in [Fol75].

Definition 2.1. A function $f: \mathbb{H}^{1} \rightarrow \mathbb{C}$ is said to be homogeneous of degree $\lambda$ (here $\lambda \in \mathbb{C}$ ) if $f \circ \gamma_{r}=r^{\lambda} f$. A distribution $\tau$ on $\mathbb{H}^{1}$ is said to be homogeneous of degree $\lambda$ if for every $u \in C_{0}^{\infty}$ and every $r>0$,

$$
\left\langle\tau, u \circ \gamma_{r}\right\rangle=r^{-Q-\lambda}\langle\tau, u\rangle
$$

If $\tau$ is given by integration against an $L_{\text {loc }}^{1}$ function $f$, these two definitions agree.

Definition 2.2. A distribution which is homogeneous of degree $\lambda-Q$ and which is $C^{\infty}$ away from 0 will be called a kernel of type $\lambda$. The vector space of kernels of type $\lambda$ will be denoted by $\mathcal{O}_{\lambda}$.

We will only be concerned with kernels of type $\lambda$, where $\lambda \geq 0$. We therefore restrict our attention to these operators. One may topologize $\mathcal{O}_{\lambda}$ in the following way: If $\lambda>0$ we identify $K \in \mathcal{O}_{\lambda}$ with the function $f \in C^{\infty}\left(\mathbb{H}^{1} \backslash\{0\}\right)$ that it agrees with away from 0 . We restrict this function to $1 \leq|\zeta| \leq 2$ (which uniquely defines the distribution by homogeneity) and therefore can identify $\mathcal{O}_{\lambda}$ with a subset of $C^{\infty}(1 \leq|\zeta| \leq 2)$, and give $\mathcal{O}_{\lambda}$ the subspace topology. When $\lambda=0$, we do the same thing, but now we have to account for the fact that $K=P V(f)+C \delta$ (here, $P V$ stands for the integral taken in the principle value sense, see [Fol75]). Thus, we identify $K$ with $\left(\left.f\right|_{1 \leq|\zeta| \leq 2}, C\right) \in C^{\infty}(1 \leq|\zeta| \leq 2) \times \mathbb{C}$. Again we give $\mathcal{O}_{\lambda}$ the subspace topology. In either case, $\mathcal{O}_{\lambda}$ becomes a Fréchet space. We will only use this topology briefly in the sequel.

For $K \in \mathcal{O}_{\lambda}$ and $u \in C_{0}^{\infty}\left(\mathbb{H}^{1}\right)$, we may define

$$
\mathrm{Op}_{L}(K) u=u * K
$$

Here, $u * K$ is the group convolution. We choose the notation $\mathrm{Op}_{L}$ since this is a left invariant operator. We will also define $\mathrm{Op}_{R}(K) u=K * u$. We will write $\mathrm{Op}_{L}\left(\mathcal{O}_{\alpha}\right)$ for the vector space $\left\{\mathrm{Op}_{L}(K): K \in \mathcal{O}_{\alpha}\right\}$, and topologize this space so that $\mathrm{Op}_{L}$ is a homeomorphism. We define $\mathrm{Op}_{R}\left(\mathcal{O}_{\alpha}\right)$ in a similar manner. All of the results of this section work equally well with $\mathrm{Op}_{R}$ replacing $\mathrm{Op}_{L}$, provided one replaces $X_{L}$ and $Y_{L}$ with their right invariant analogs. For convenience (for the rest of this paper), we will denote by $L^{(\alpha)}$ an arbitrary element of $\mathrm{Op}_{L}\left(\mathcal{O}_{\alpha}\right)$, and by $R^{(\alpha)}$ an arbitrary element of $\mathrm{Op}_{R}\left(\mathcal{O}_{\alpha}\right)$. The following propositions are well known, and mostly contained in [Fol75].

Proposition 2.3. Suppose $0 \leq \lambda<Q, 1<p<\frac{Q}{\lambda}$, and $\frac{1}{q}=\frac{1}{p}-\frac{\lambda}{Q}$. Then, $L^{(\lambda)}$ extends to a bounded operator $L^{p} \rightarrow L^{q}$, which we will again denote by $L^{(\lambda)}$.

Proposition 2.4. Suppose $0 \leq \alpha, \beta, \alpha+\beta<Q$, and $K_{\alpha} \in \mathcal{O}_{\alpha}, K_{\beta} \in \mathcal{O}_{\beta}$. Then,

$$
\mathrm{Op}_{L}\left(K_{\alpha}\right) \mathrm{Op}_{L}\left(K_{\beta}\right)=\mathrm{Op}_{L}\left(K_{\alpha+\beta}\right)
$$

where $K_{\alpha+\beta} \in \mathcal{O}_{\alpha+\beta}$. Also, if $\phi \prec \psi$ are nested $C_{0}^{\infty}$ functions, then

$$
\phi \mathrm{Op}_{L}\left(K_{\alpha}\right) \psi \mathrm{Op}_{L}\left(K_{\beta}\right) \equiv \phi \mathrm{Op}_{L}\left(K_{\alpha}\right) \mathrm{Op}_{L}\left(K_{\beta}\right)
$$


Proposition 2.5. $X_{L}$ and $Y_{L}$ map $\mathrm{Op}_{L}\left(\mathcal{O}_{\alpha}\right) \rightarrow \mathrm{Op}_{L}\left(\mathcal{O}_{\alpha-1}\right)$ continuously provided $\alpha>1$. Moreover, for $K_{\alpha} \in \mathcal{O}_{\alpha}, X_{L} \mathrm{Op}\left(K_{\alpha}\right)=\mathrm{Op}\left(X_{L} K_{\alpha}\right)$, similarly for $Y_{L}$.

Proposition 2.6. If we define $\mathcal{L}_{0}^{(L)}=X_{L}^{2}+Y_{L}^{2}$ (the so-called sublaplacian), then $\mathcal{L}_{0}^{(L)}$ has a fundamental solution $\mathcal{J} \in \mathrm{Op}_{L}\left(\mathcal{O}_{2}\right) . \quad I e, \mathcal{L}_{0}^{(L)} \mathcal{J}=\mathcal{J}_{\mathcal{L}_{0}^{(L)}}=I$ as operators on $C_{0}^{\infty}$.

Remark 2.7. We used the notation $\mathcal{L}_{0}^{(L)}$ in Proposition 2.6 since we will define more general operators in Section 2.3.

An easy consequence of Proposition 2.6 is the following:

Proposition 2.8. Suppose $T \in \mathrm{Op}_{L}\left(\mathcal{O}_{j}\right)(j<4)$. Then, there exist $T_{1}, T_{2} \in$ $\mathrm{Op}_{L}\left(\mathcal{O}_{j}\right)$ such that:

$$
X_{L} T=T_{1} X_{L}+T_{2} Y_{L}
$$

a similar result holds for $Y_{L} T$.

2.1.1. Operators of Rothschild and Stein. We will now need to review a certain class of operators from [RS76] due to Rothschild and Stein (though what we introduce here will not be their operators in full generality). The operators below are also special cases of those covered in [CGGP92].

Definition 2.9. A distribution kernel $K(\xi, \eta)$ on $\mathbb{H}^{1} \times \mathbb{H}^{1}$ is said to be a pseudodifferential kernel of type $\lambda, \lambda \geq 0$, if for any positive integer $l$, we can write:

$$
K(\xi, \eta)=\sum_{i=1}^{s} a_{i}(\xi) K_{\xi}^{(i)}\left(\eta^{-1} \xi\right) b_{i}(\eta)+E_{l}(\xi, \eta)
$$

where,

- $E_{l} \in C_{0}^{l}\left(\mathbb{H}^{1} \times \mathbb{H}^{1}\right)$,

- $a_{i}, b_{i} \in C_{0}^{\infty}\left(\mathbb{H}^{1}\right), i=1, \ldots, s$,

- For each $i, \xi \mapsto K_{\xi}^{(i)}$ is a smooth map $\mathbb{H}^{1} \rightarrow \mathcal{O}_{\alpha_{i}}$ for some $\alpha_{i} \geq \lambda$.

And we call an operator whose distribution kernel is a pseudodifferential kernel of type $\lambda$, a pseudodifferential convolution operator of type $\lambda$.

Remark 2.10. Note that, in light of the definition, the distribution $K$ has compact support in $\mathbb{H}^{1} \times \mathbb{H}^{1}$. Also, it is clear from the definition that if $T$ is a pseudodifferential convolution operator of type $\lambda \geq 1$, then $X_{L} T$ and $Y_{L} T$ are pseudodifferential convolution operators of type $\lambda-1$.

It is shown in [RS76] that the class of pseudodifferential kernels of type $\lambda$ is closed under adjoints. We will review the regularity properties of such operators in Section 2.4. From the calculus of [CGGP92], we easily see:

Proposition 2.11. Suppose $T$ is a pseudodifferential convolution operator of type $\lambda$, and $f \in C_{0}^{\infty}$. Then, $[T, f]$ is a pseudodifferential convolution operator of type $\lambda+1$.

Proof. One can either use the calculus of [CGGP92] or see the bottom of page 568 of [CG84] for a direct proof.

Example 2.12. In this example, we will discuss the argument in the proof of Theorem 10 of [RS76]. Suppose for $\alpha \in \mathbb{R}^{n}, P_{\alpha}\left(X_{L}, Y_{L}\right)$ is a homogeneous noncommutative polynomial of degree $m$ that is hypoelliptic and depends smoothly on $\alpha$ 
(namely its coefficients depend smoothly on $\alpha$, and we think of the coefficients as being to the left of the vector fields). Since $P_{\alpha}\left(X_{L}, Y_{L}\right)$ is hypoelliptic, it has a fundamental solution in $\phi_{\alpha} \in \mathcal{O}_{m}$. Suppose, further, that $\alpha \mapsto \phi_{\alpha}$ is a smooth map $\mathbb{R}^{n} \rightarrow \mathcal{O}_{m}$. If $\beta: \mathbb{H}^{1} \rightarrow \mathbb{R}^{n}$ is smooth, and $\psi, \psi^{\prime} \in C_{0}^{\infty}$ we may define the integral kernel:

$$
K(\xi, \eta)=\psi(\xi) \phi_{\beta(\xi)}\left(\eta^{-1} \xi\right) \psi^{\prime}(\eta)
$$

If we consider $X_{L}$ and $Y_{L}$ acting in the $\xi$ variable, and we consider the equation:

$$
P_{\beta(\xi)}\left(X_{L}, Y_{L}\right) K(\xi, \eta)
$$

there are two types of terms. If any of the vector fields land on $\psi(\xi)$ or on $\beta(\xi)$, we are left with pseudodifferential kernel of type at least 1 . This is clear when the vector fields land on $\psi(\xi)$, so let us consider (as an example) the case when $X_{L}$ lands on $\beta(\xi)$. We are then left with the term:

$$
\psi(\xi)\left(X_{L} \beta\right)(\xi)\left(\partial_{\alpha} \phi\right)_{\beta(\xi)}\left(\eta^{-1} \xi\right) \psi^{\prime}(\eta)
$$

and this is again a pseudodifferential kernel of type $m$. Thus we may imagine all of our vector fields land on the $\xi$ of $\phi_{\beta}\left(\eta^{-1} \xi\right)$ so long as we are willing to work modulo error terms that are pseudodifferential kernels of type 1 . But, when all the vector fields land on the $\xi$ of $\phi_{\alpha}\left(\eta^{-1} \xi\right)$, we may imagine $\beta$ is fixed, and use the formula $P_{\beta}\left(X_{L}, Y_{L}\right) \phi_{\beta}=\delta_{0}$ to see that if $T$ is the operator with Schwartz kernel $K$ we have

$$
P_{\beta(\xi)}\left(X_{L}, Y_{L}\right) T=\psi \psi^{\prime}+E^{(1)}
$$

where $E^{(1)}$ is a pseudodifferential convolution operator of type 1 .

2.2. Mix of Left and Right Convolution Operators. In Section 2.1, we reviewed the properties of operators defined by

$$
f \mapsto f * K
$$

where $K \in \mathcal{O}_{\lambda}$ for some $\lambda$, yielding a left invariant convolution operator. The entire theory, there reviewed, works exactly the same on operators given by:

$$
f \mapsto K * f
$$

provided we replace our left invariant vector fields $X_{L}, Y_{L}$, with their right invariant analogs:

$$
X_{R}=\partial_{x}-2 y \partial_{t}, \quad Y_{R}=\partial_{y}+2 x \partial_{t}
$$

This then leaves us with a right invariant convolution operator. In this section, we will review the basic properties of an operator given by a composition of such a left invariant operator with a right invariant one. The author has no doubt that these results are well known, but was unable to find a suitable reference. All of the results in this section follow by standard methods, and we leave the details to the reader. Most of the results follow from the methods used in [Fol75], with slight modifications.

Remark 2.13. The results in this section are not actually necessary for our main results, since we are just constructing a local parametrix, and $R^{(j)} \phi L^{(j)}\left(\phi \in C_{0}^{\infty}\right)$ is an easy operator to understand: it is clearly pseudolocal, and its mapping properties follow from those of $R^{(j)}$ and $L^{(j)}$. However, it will be nice for some side issues and for general convenience to understand operators like $R^{(0)} L^{(0)}$. 
If $L^{(\alpha)} \in \mathrm{Op}_{L}\left(\mathcal{O}_{\alpha}\right), R^{(\beta)} \in \mathrm{Op}_{R}\left(\mathcal{O}_{\beta}\right)$ and $0 \leq \alpha, \beta, \alpha+\beta<Q$, Proposition 2.3 gives us a way to define $R^{(\beta)} L^{(\alpha)} f$ where $f \in C_{0}^{\infty}$. Indeed, suppose $p>1$ but so small that $\frac{1}{p}-\frac{\beta+\alpha}{Q}>0$. Then if $q=\left(\frac{1}{p}-\frac{\beta+\alpha}{Q}\right)^{-1}$, we see that $R^{(\beta)} L^{(\alpha)}$ is a bounded map from $L^{p}$ to $L^{q}$. The results we need about $R^{(\beta)} L^{(\alpha)}$ are summarized below:

- $R^{(\beta)}$ and $L^{(\alpha)}$ commute. That is, $R^{(\beta)} L^{(\alpha)}=L^{(\alpha)} R^{(\beta)}$.

- $R^{(\beta)} L^{(\alpha)}$ is pseudolocal.

- If $\phi \prec \psi \in C_{0}^{\infty}$, then $\phi R^{(\beta)} L^{(\alpha)} \equiv \phi R^{(\beta)} \psi L^{(\alpha)}$.

- If $\phi \prec \psi \in C_{0}^{\infty}$, then $\phi R^{(\beta)}(1-\psi) L^{(\alpha)} \equiv 0$.

- If $\phi, \psi \in C_{0}^{\infty}$ have disjoint supports, then $\phi R^{(\beta)} \psi L^{(\alpha)} \equiv 0$.

- $X_{L}$ and $Y_{L}$ commute with $R^{(\beta)}$, while $X_{R}$ and $Y_{R}$ commute with $L^{(\alpha)}$.

Remark 2.14. Suppose $L_{1}^{(0)}, L_{2}^{(0)} \in \mathrm{Op}_{L}\left(\mathcal{O}_{0}\right)$ and $R_{1}^{(0)}, R_{2}^{(0)} \in \mathrm{Op}_{R}\left(\mathcal{O}_{0}\right)$, then if $\psi \prec \phi$,

$$
\begin{aligned}
\psi L_{1}^{(0)} R_{1}^{(0)} \phi L_{2}^{(0)} R_{2}^{(0)} \phi & \equiv \psi L_{1}^{(0)} \phi R_{1}^{(0)} \phi L_{2}^{(0)} \phi R_{2}^{(0)} \phi \\
& \equiv \psi L_{1}^{(0)} \phi R_{1}^{(0)} L_{2}^{(0)} \phi R_{2}^{(0)} \phi \\
& \equiv \psi L_{1}^{(0)} L_{2}^{(0)} \phi R_{1}^{(0)} R_{2}^{(0)} \phi
\end{aligned}
$$

The same thing would happen if we replaced, say, $R_{2}^{(0)}$ with $R_{2}^{(\alpha)} \in \mathrm{Op}_{R}\left(\mathcal{O}_{\alpha}\right)$, so long as $\alpha<Q$.

2.3. Some Special Operators. In the beginning of this section, we introduced the left invariant vector fields $Z_{L}$ and $\bar{Z}_{L}$. Following [Ste93], we define, for $\alpha \in \mathbb{C}$,

$$
\begin{aligned}
\mathcal{L}_{\alpha}^{(L)} & =-\frac{1}{2}\left(Z_{L} \bar{Z}_{L}+\bar{Z}_{L} Z_{L}+\alpha\left[Z_{L}, \bar{Z}_{L}\right]\right) \\
& =-\left(Z_{L} \bar{Z}_{L}+\frac{1}{2}(\alpha-1)\left[Z_{L}, \bar{Z}_{L}\right]\right)
\end{aligned}
$$

Essentially all the results in this section can be found in [Ste93], but many were introduced in [FS74]. We state the results we need without proof, leaving the details to the reader. When $\alpha$ is not an odd integer (here an odd integer can be either positive or negative), $\mathcal{L}_{\alpha}^{(L)}$ has a fundamental solution given by:

$$
\theta_{\alpha}(z, t)=\frac{\Gamma\left(\frac{1+\alpha}{2}\right) \Gamma\left(\frac{1-\alpha}{2}\right)}{2 \pi^{2}}\left(|z|^{2}-i t\right)^{-(1+\alpha) / 2}\left(|z|^{2}+i t\right)^{-(1-\alpha) / 2}
$$

Ie, $\mathcal{L}_{\alpha}^{(L)} \theta_{\alpha}=\delta_{0}$. Note that $\theta_{\alpha} \in \mathcal{O}_{2}$. Moreover, it is clear from Equation (2.1) that the map $\alpha \mapsto \mathcal{O}_{2}$ is meromorphic in $\alpha$, and has simple poles at the odd integers.

For $n$ an odd integer, $\mathcal{L}_{n}^{(L)}$ has a nontrivial $L^{2}$ kernel; let $\pi_{n}^{(L)}$ denote the selfadjoint projection onto the $L^{2}$ kernel of $\mathcal{L}_{n}^{(L)} . \pi_{n}^{(L)} \in \mathrm{Op}_{L}\left(\mathcal{O}_{0}\right)$ and, moreover, $\pi_{n}^{(L)}=D_{t} L^{(2)}$ (for some $L^{(2)} \in \mathrm{Op}_{L}\left(\mathcal{O}_{2}\right)$, depending on $n$ ). Also, there exists an operator $P_{n}^{(L)} \in \mathrm{Op}_{L}\left(\mathcal{O}_{2}\right)$ such that $P_{n}^{(L)} \mathcal{L}_{n}^{(L)}=1-\pi_{n}^{(L)}=\mathcal{L}_{n}^{(L)} P_{n}^{(L)}$. Finally,

$$
\left[\mathcal{L}_{\beta}^{(L)}, \mathcal{L}_{\alpha}^{(L)}\right]=0
$$

for all $\alpha, \beta \in \mathbb{C}$,

$$
\left[\mathcal{L}_{\alpha}^{(L)}, P_{n}^{(L)}\right]=0=\left[\mathcal{L}_{\alpha}^{(L)}, \pi_{n}^{(L)}\right]
$$


for all $\alpha \in \mathbb{C}$ and $n$ an odd integer, and

$$
\left[P_{n_{1}}^{(L)}, P_{n_{2}}^{(L)}\right]=0=\left[P_{n_{1}}^{(L)}, \pi_{n_{2}}^{(L)}\right]
$$

for all $n_{1}, n_{2}$ odd integers.

Since it will be of great important to us, we fix the notation $\pi^{(L)}=\pi_{1}^{(L)}$ and $P^{(L)}=P_{1}^{(L)}$. All of the results of this section move over without change to the right invariant vector fields $Z_{R}, \bar{Z}_{R}$ (defined by $\bar{Z}_{R}=\frac{1}{2}\left(X_{R}+i Y_{R}\right)$ and similarly for $\left.Z_{R}\right)$. Really, these results are related to the others by the change of variables $t \rightarrow-t$. Whence, we have $P_{n}^{(R)} \in \mathrm{Op}_{R}\left(\mathcal{O}_{2}\right)$ and $\pi_{n}^{(R)} \in \mathrm{Op}_{R}\left(\mathcal{O}_{0}\right)$ satisfying $\mathcal{L}_{n}^{(R)} P_{n}^{(R)}=1-\pi_{n}^{(R)}$.

It is well known, and easily seen, that the range of $\pi_{1}^{(L)}$ is supported on $D_{t}>0$ and the range of $\pi_{n}^{(R)}(n>0)$ is supported on $D_{t}<0$. Thus, $\pi_{1}^{(L)} \pi_{n}^{(R)}=0$ for every odd $n>0$.

2.4. Degrees of Smoothing. The $L^{p}$ regularity properties of pseudodifferential convolution operators, and of left invariant convolution operators, is best understood in terms of nonisotropic $L^{p}$ Sobolev spaces, which we now define. Definitions of these spaces (and background on them) can be found in [Fol75, Koe02, NS79, RS76] and many other sources.

We let $\nabla_{L}=\left(X_{L}, Y_{L}\right)$. We use the standard notation of $\nabla_{L}^{I}$, where $I$ denotes a sequences of $1 \mathrm{~s}$ and $2 \mathrm{~s}$ (an ordered multi-index, see Section 1.2). $|I|$ denotes the length of the sequence. The non-isotropic Sobolev spaces $N L_{k}^{p}($ for $k \in \mathbb{N}$ ) are given by the completion of $C_{0}^{\infty}\left(\mathbb{H}^{1}\right)$ in the norm

$$
\|u\|_{N L_{k}^{p}}=\sum_{|I| \leq k}\left\|\nabla_{L}^{I} u\right\|_{L^{p}}
$$

One may define $N L_{s}^{p}$ for $0 \leq s \in \mathbb{R}$ by complex interpolation (see [Fol75] for a detailed account) and all of our results extend to these spaces (by interpolation), but we will not bother mentioning such details.

Definition 2.15. Suppose $T$ is an operator taking $C_{0}^{\infty}$ to distributions. We say that $T$ is smoothing of order $\lambda^{-}(\lambda \geq 0)$ if $T$ is pseudolocal and:

(1) $T$ extends to a continuous map $N L_{k, \mathrm{cpt}}^{p} \rightarrow N L_{k+m, \text { loc }}^{p}$, for every $k \geq 0$ where $0 \leq m \leq \lambda$ is an integer and $1<p<\infty$.

(2) $T$ extends to a continuous map $L_{s, \mathrm{cpt}}^{p} \rightarrow L_{s+\lambda / 2 \text {,loc }}^{p}$, for every $s \in \mathbb{R}$ and every $1<p<\infty$

(3) $T$ extends to a continuous map $\Lambda_{\alpha, \mathrm{cpt}} \rightarrow \Lambda_{\alpha-\epsilon+\lambda / 2 \text {,loc }}$, for every $\epsilon>0$ and every $\alpha>0$ with $\alpha>\epsilon-\lambda / 2$.

(4) There exist $S_{1}, S_{2}$, and $S_{3}$ smoothing of order $\lambda^{-}$(this definition is recursive) such that $X_{L} T=S_{1} X_{L}+S_{2} Y_{L}+S_{3}$. Similarly for $Y_{L} T$.

(5) If $\lambda \geq 1$ then $X_{L} T, Y_{L} T, T X_{L}$, and $T Y_{L}$ are smoothing of order $(\lambda-1)^{-}$.

In the above definition, the - in $\lambda^{-}$really just refers to the available smoothing in Lipschitz spaces. These are so defined because $L^{(0)}$ is smoothing of order $0^{-}$in this sense. $L^{(j)}$ for $j>0$ is better than smoothing of order $j^{-}$, though, so we make a definition to accommodate that:

Definition 2.16. Suppose $T$ is an operator taking $C_{0}^{\infty}$ to distributions. We say that $T$ is smoothing of order $\lambda(\lambda \geq 0)$ if $T$ is pseudolocal and: 
(1) $T$ extends to a continuous map $N L_{k, \mathrm{cpt}}^{p} \rightarrow N L_{k+m, \text { loc }}^{p}$, for every $k \geq 0$ where $0 \leq m \leq \lambda$ is an integer and $1<p<\infty$.

(2) $T$ extends to a continuous map $L_{s, \mathrm{cpt}}^{p} \rightarrow L_{s+\lambda / 2 \text {, loc }}^{p}$, for every $s \in \mathbb{R}$ and every $1<p<\infty$.

(3) $T$ extends to a continuous map $\Lambda_{\alpha, \mathrm{cpt}} \rightarrow \Lambda_{\alpha+\lambda / 2 \text {, loc }}$, for every $\alpha>0$.

(4) There exist $S_{1}, S_{2}$, and $S_{3}$ smoothing of order $\lambda$ such that $X_{L} T=S_{1} X_{L}+$ $S_{2} Y_{L}+S_{3}$. Similarly for $Y_{L} T$.

(5) If $\lambda>1$ then $X_{L} T, Y_{L} T, T X_{L}$, and $T Y_{L}$ are smoothing of order $\lambda-1$.

(6) If $\lambda=1$ then $X_{L} T$ and $Y_{L} T$ are smoothing of order $0^{-}$.

We state some results that will be used in the sequel, and are either contained in [RS76], or easy to prove given the results in [RS76] and [NS79].

Proposition 2.17. Suppose $T$ is smoothing of order $\alpha$ and $S$ is smoothing of order $\beta$, then if $\phi \in C_{0}^{\infty}, T \phi S$ is smoothing of order $\alpha+\beta$. If, instead, $T$ is smoothing of order $\alpha^{-}$or $S$ is smoothing of order $\beta^{-}$(or both) then T $\phi S$ is smoothing of order $(\alpha+\beta)^{-}$.

Proposition 2.18. Suppose $T$ is a pseudodifferential convolution operator of type $j>0$, then $T$ is smoothing of order $j$. If, instead, $T$ is a pseudodifferential convolution operator of type 0 , then $T$ is smoothing of order $0^{-}$.

Proposition 2.19. $D_{t} R^{(1)} L^{(1)}$ is smoothing of order 0 .

Corollary 2.20. $R^{(1)} L^{(0)} \pi^{(L)}$ is smoothing of order 1 .

Proposition 2.21. $D_{t} R^{(2)}$ is smoothing of order 0 and $R^{(0)}$ is smoothing of order $0^{-}$.

Remark 2.22. We restate here a remark from page 82 of [NS79]. Suppose $T^{(0)}$ is a pseudodifferential convolution operator of type 0 . Proposition 2.18 only guarantees that $T^{(0)}$ is smoothing of order $0^{-}$. However, if $T^{(0)}=D_{t} T^{(2)}$, then $T^{(0)}$ is smoothing of order 0 by the fact that $T^{(2)}$ is smoothing of order 2 . In particular, the fact that $\pi^{(L)}=D_{t} L^{(2)}$ implies that $\pi^{(L)}$ is smoothing of order 0 (see Section $2.3)$.

\section{The Parametrix}

Recall the operator $\mathcal{A}$ from Section 1.1 (and the operators $A_{j}, j=1, \ldots, 6$ ). Note that by conjugating $\mathcal{A}$ by the change of variables $t \mapsto t+t_{0}$, we may (wlog) assume $t_{0}=0$. We will, henceforth, make this assumption, and construct a left parametrix for $\mathcal{A}$, near $0 \in \mathbb{H}^{1}$.

Remark 3.1. By conjugating $\mathcal{A}$ by the translation given by left multiplication by $\left(z_{0}, 0\right)$, one can use our results to describe loss near $z=z_{0}$ instead of $z=0$. See Example 5.1.

Remark 3.2. Without increasing the class of operators covered by $\mathcal{A}$, we see that we could have included terms like:

$$
A_{7}=\left(\sum_{\substack{d+e=a+b+1 \\ 0 \leq d, e}} z^{d} \bar{z}^{e} g_{d, e}^{(5)}(z, t)\right) D_{t}
$$


Indeed, writing $D_{t}=\frac{1}{2}\left[Z_{L}, \bar{Z}_{L}\right]$, it is easy to see that

$$
\sum_{j=1}^{7} A_{j}
$$

is of the form

$$
f \sum_{j=1}^{6} A_{j}^{\prime}
$$

where $f$ is a $C^{\infty}$ function that is nonzero at 0 .

Many of the technical difficulties come from the $A_{6}$ term. Indeed, the parametrix construction is far easier when $A_{6}=0$, and when $A_{6} \neq 0$ and is of the general form above, we have to use $C^{L}$ functions in our construction (instead of only $C^{\infty}$ functions), where $L$ is any fixed integer we choose. This does not affect our regularity results, as we may pick an $L$ large enough for any regularity result we wish to prove, but does make the construction much less elegant. We, therefore, proceed with the construction under the assumption $A_{6}=0$, and we will return to the more general case in Section 3.4.

3.1. Operators Which Vanish At 0. This section is devoted to proving the following result:

Theorem 3.3. Let $\phi \prec \phi^{\prime}$ be a cut off functions with support sufficiently close to $0 \in \mathbb{H}^{1}$. Then there exists a pseudodifferential convolution operator $U^{(2)}$ of type 2 , such that:

$$
U^{(2)} \mathcal{A}=\phi\left(1-\pi^{(L)}\right) \phi^{\prime}+\kappa^{(1)}
$$

where $\kappa^{(1)}$ is a pseudodifferential convolution operator of type 1.

In this section, $A_{3}, A_{4}$, and $A_{5}$ will be considered as lower order terms. Define $\mathcal{A}_{0}=A_{1}+A_{2}$. Note that if we have Theorem 3.3 with $\mathcal{A}_{0}$ in place of $\mathcal{A}$, then we have the theorem for $\mathcal{A}$. Thus, we proceed with proving Theorem 3.3 with $\mathcal{A}_{0}$ in place of $\mathcal{A}$. Define

so that

$$
F(z, t)=z^{a} \bar{z}^{b} h(z, t)
$$

$$
\mathcal{A}_{0}=Z_{L} \bar{Z}_{L}+\bar{Z}_{L} F(\zeta) Z_{L}
$$

Proposition 3.4. Suppose $\phi$ is a cut off function supported sufficently close to $0 \in \mathbb{H}^{1}$. Then, there exists a pseudodifferential convolution operator $S^{(2)}$, of type 2 , such that

$$
S^{(2)} \mathcal{A}_{0}=F(\zeta) \phi+\kappa^{(1)}
$$

where $\kappa^{(1)}$ is a pseudodifferential convolution operator of type 1.

Proof. Consider, without worrying about dividing by zero for the moment,

$$
\begin{aligned}
\mathcal{A}_{0} & =Z_{L} \bar{Z}_{L}+\bar{Z}_{L} F(\zeta) Z_{L} \\
& =(1+F(\zeta)) Z_{L} \bar{Z}_{L}-F(\zeta)\left[Z_{L}, \bar{Z}_{L}\right]+\left(\left(\bar{Z}_{L} F\right)(\zeta)\right) Z_{L} \\
& =-(1+F(\zeta)) \mathcal{L}_{\beta(\zeta)}^{(L)}+\left(\left(\bar{Z}_{L} F\right)(\zeta)\right) Z_{L}
\end{aligned}
$$

where

$$
\beta(\zeta)=\frac{1-F(\zeta)}{1+F(\zeta)}
$$


Note that $\beta(0)=1$, and so $\beta(\zeta)$ is as close to 1 as we like, so long as we keep $\zeta$ close to 0 .

Recall $\theta_{\alpha}$ from (2.1). $\theta_{\alpha}$ is meromorphic in $\alpha$ with a simple pole at $\alpha=1$ and satisfies

$$
\mathcal{L}_{\alpha}^{(L)} \theta_{\alpha}=\delta_{0}
$$

Thus, $(1-\alpha) \theta_{\alpha}$ is a holomorphic map $\alpha \rightarrow \mathcal{O}_{2}$ for $\alpha$ near 1 .

Let $\phi \prec \phi^{\prime} \in C_{0}^{\infty}$ equal 1 on a neighborhood of $0 \in \mathbb{H}^{1}$, and 0 outside a small neighborhood of 0 . Namely, we wish the neighborhood to be so small that $(1-\alpha) \theta_{\alpha}$ is holomorphic where $\alpha$ ranges over a neighborhood of $\beta\left(\operatorname{supp}\left(\phi^{\prime}\right)\right)$, and $F(\zeta)$ stays away from -1 on $\operatorname{supp}\left(\phi^{\prime}\right)$. Whence, on the support of $\phi^{\prime}$, our considerations in (3.1) become rigorous.

Define the kernel $K(\xi, \eta)$ (mapping from the $\eta$ variable to the $\xi$ variable) by

$$
K(\xi, \eta)=\phi(\xi)(1-\beta(\eta)) \theta_{\beta(\eta)}\left(\eta^{-1} \xi\right) \phi^{\prime}(\eta) \frac{-1}{1+F(\eta)}
$$

Let $T^{(2)}$ be the pseudodifferential convolution operator of type 2 with kernel $K$. By an argument similar to that in Example 2.12 we see that:

$$
T^{(2)} \mathcal{A}_{0}=\phi(1-\beta)+E^{(1)}
$$

where $E^{(1)}$ is a pseudodifferential convolution operator of type 1. But,

$$
\begin{aligned}
1-\beta(\xi) & =\left(1-\frac{1-F(\xi)}{1+F(\xi)}\right) \\
& =\frac{2 F(\xi)}{1+F(\xi)}
\end{aligned}
$$

Thus, taking $S^{(2)}=\frac{1+F(\xi)}{2} T^{(2)}$, the claim follows.

Remark 3.5. We can now easily construct a parametrix for Kohn's operator away from $z=0$. Indeed, for Kohn's operator, $F(\zeta)=|z|^{2 k}$, and so by Equation (3.1), we see: $\mathcal{A}=-(1+F(\zeta)) \mathcal{L}_{\beta(\zeta)}^{(L)}+\left(\left(\bar{Z}_{L} F\right)(\zeta)\right) Z_{L}$. Where,

$$
\beta(\zeta)=\frac{1-|z|^{2 k}}{1+|z|^{2 k}}
$$

and so $-1<\beta(\zeta)<1$ for $z \neq 0$. Thus, if we are concerned with regularity near a point $0 \neq \xi_{0} \in \mathbb{H}^{1}$, we let $\psi \in C_{0}^{\infty}$ be a bump function that is 1 on a neighborhood of $\xi_{0}$ and 0 on a neighborhood of 0 and we let $S$ be the operator with kernel (here, $\eta=(w, s))$ :

$$
K(\xi, \eta)=\psi(\xi) \theta_{\beta(\eta)}\left(\eta^{-1} \xi\right) \frac{1}{1+|w|^{2 k}} \psi(\eta)
$$

and then by the argument in [RS76] (see Example 2.12) $S$ will be locally (near $\xi_{0}$ ) a parametrix for $\mathcal{A}$, and $S$ is a pseudodifferential convolution operator of type 2 . This proves maximal regularity in $L^{p}$ and Lipschitz spaces (see [RS76]; this operator fits exactly into that framework for $z$ away from 0 ). Because of this, we say that Kohn's operator is "maximally hypoelliptic" away from $z=0$.

Remark 3.6. Proposition 3.4 implies that $F(\zeta)$ is a "subelliptic multiplier." 
We now turn to proving Theorem 3.3. Let $\phi \prec \phi^{\prime}$ be nested cut off functions (of small support near $0 \in \mathbb{H}^{1}$ ), where $\phi^{\prime}$ plays the role of $\phi$ in Proposition 3.4. Define:

$$
U^{(2)}=\phi P^{(L)} \phi^{\prime}-\phi P^{(L)} \bar{Z}_{L} Z_{L} S^{(2)}
$$

Then, consider, (here $\kappa^{(1)}$ will be a pseudodifferential convolution operator of type 1 that may change from line to line)

$$
\begin{aligned}
U^{(2)} \mathcal{A}_{0} & =\phi P^{(L)} A_{1} \phi^{\prime}+\phi P^{(L)} A_{2} \phi^{\prime}-\phi P^{(L)} \bar{Z}_{L} Z_{L} \phi^{\prime} F(\zeta)+\kappa^{(1)} \\
& =\phi\left(1-\pi^{(L)}\right) \phi^{\prime}+\phi P^{(L)} \bar{Z}_{L} Z_{L} F(\zeta) \phi^{\prime}-\phi P^{(L)} \bar{Z}_{L} Z_{L} \phi^{\prime} F(\zeta)+\kappa^{(1)} \\
& =\phi\left(1-\pi^{(L)}\right) \phi^{\prime}+\kappa^{(1)}
\end{aligned}
$$

which completes the proof of Theorem 3.3.

Remark 3.7. The astute reader may note that $U^{(2)}$ is really the composition of two pseudodifferential convolution operators, one of type 2 and one of type 0 . Indeed, this is again a pseudodifferential convolution operator, as claimed. This can be seen by the calculus of [CGGP92]. We do not really need this result however, since all we really need is that $U^{(2)}$ is smoothing of order 2 . This comes from the fact that $P^{(L)} \bar{Z}_{L} Z_{L}=-2 D_{t} P^{(L)}+1-\pi^{(L)}$, and therefore is smoothing of order 0 . Alternatively, one could see these operators in the calculus of [NS79], and use the composition results there.

3.2. Calculation of Certain Operators. In light of Theorem 3.3, it suffices to find an operator $T$ such that:

$$
T \mathcal{A}=\phi \pi^{(L)} \phi^{\prime}+E^{\left(1^{-}\right)}
$$

Where $E^{\left(1^{-}\right)}$is smoothing of order $1^{-}$. Let $A_{2}^{\prime}=\bar{Z}_{L} z^{a} \bar{z}^{b} Z_{L}$. Ie, $A_{2}^{\prime}$ is like $A_{2}$, except with $h=1$. We will achieve Equation 3.2 by constructing an $\mathcal{M}$ such that:

- $\mathcal{M} \pi^{(L)}$ is pseudolocal

- $\mathcal{M} \pi^{(L)} A_{2}^{\prime} \pi^{(L)}=\pi^{(L)}$

- $\mathcal{M} \pi^{(L)} A_{2}^{\prime}$ is smoothing of order 0

- $\mathcal{M} \pi^{(L)}\left(A_{3}+A_{4}+A_{5}\right)$ is smoothing of order $1^{-}$

Where we must show that these operators even make sense. For a moment, let's suppose we have such an $\mathcal{M}$, and show how this will formally yield Equation 3.2, while not yet worrying whether or not our computations make sense. Let $\phi \prec \phi^{\prime}$ be nested $C^{\infty}$ functions which are 1 on a neighborhood of $0 \in \mathbb{H}^{1}$.

$$
\begin{aligned}
\frac{1}{h(\zeta)} \phi \mathcal{M} \pi^{(L)} \phi^{\prime} \mathcal{A} \equiv & \frac{1}{h(\zeta)} \phi \mathcal{M} \pi^{(L)} A_{2} \phi^{\prime}+E^{\left(1^{-}\right)} \\
= & \frac{1}{h(\zeta)} \phi \mathcal{M} \pi^{(L)} \bar{Z}_{L} z^{a} \bar{z}^{b} Z_{L} \phi^{\prime} h(\zeta) \\
& +\frac{1}{h(\zeta)} \phi \mathcal{M} \pi^{(L)} \bar{Z}_{L} z^{a} \bar{z}^{b}\left[h(\zeta), Z_{L}\right] \phi^{\prime}+E^{\left(1^{-}\right)}
\end{aligned}
$$

Here, we have used that we may commute $\mathcal{A}$ past $\phi^{\prime}$ with errors that are in $E^{\left(1^{-}\right)}$. Indeed, if any of the derivatives land on $\phi^{\prime}$, we use the fact that $\phi \prec \phi^{\prime}$, and that $\mathcal{M} \pi^{(L)}$ is pseudolocal, to see that the error term is infinitely smoothing. Now,

$$
\bar{Z}_{L} z^{a} \bar{z}^{b}\left[h(\zeta), Z_{L}\right]
$$


is of the form covered by $A_{4}+A_{5}$ and so we will have that

$$
\mathcal{M} \pi^{(L)} \bar{Z}_{L} z^{a} \bar{z}^{b}\left[h(\zeta), Z_{L}\right]
$$

is smoothing of order $1^{-}$as well. Hence,

$$
\begin{aligned}
& \frac{1}{h(\zeta)} \phi \mathcal{M} \pi^{(L)} \phi^{\prime} \mathcal{A}=\frac{1}{h(\zeta)} \phi \mathcal{M} \pi^{(L)} \bar{Z}_{L} z^{a} \bar{z}^{b} Z_{L} \phi^{\prime} h(\zeta)+E^{\left(1^{-}\right)} \\
& \quad=\frac{1}{h(\zeta)} \phi \mathcal{M} \pi^{(L)} A_{2}^{\prime} \pi^{(L)} \phi^{\prime} h(\zeta)+\frac{1}{h(\zeta)} \phi \mathcal{M} \pi^{(L)} A_{2}^{\prime}\left(1-\pi^{(L)}\right) \phi^{\prime} h(\zeta)+E^{\left(1^{-}\right)} \\
& \quad=\frac{1}{h(\zeta)} \phi \pi^{(L)} \phi^{\prime} h(\zeta)+\frac{1}{h(\zeta)} \phi \mathcal{M} \pi^{(L)} A_{2}^{\prime}\left(1-\pi^{(L)}\right) \phi^{\prime} h(\zeta)+E^{\left(1^{-}\right)}
\end{aligned}
$$

But if $\phi^{\prime} \prec \psi$, we know from Proposition 2.11 that $\left[\phi \pi^{(L)} \phi^{\prime}, \psi h\right]$ is smoothing of order 1 , and therefore,

$$
\begin{gathered}
\frac{1}{h(\zeta)} \phi \mathcal{M} \pi^{(L)} \phi^{\prime} \mathcal{A}=\phi \pi^{(L)} \phi^{\prime}+\frac{1}{h(\zeta)} \phi \mathcal{M} \pi^{(L)} A_{2}^{\prime}\left(1-\pi^{(L)}\right) \phi^{\prime} h(\zeta)+E^{\left(1^{-}\right)} \\
=\phi \pi^{(L)} \phi^{\prime}+\frac{1}{h(\zeta)} \phi \mathcal{M} \pi^{(L)} A_{2}^{\prime} \psi\left(1-\pi^{(L)}\right) \psi \phi^{\prime} h(\zeta)+E^{\left(1^{-}\right)} \\
=\phi \pi^{(L)} \phi^{\prime}+\frac{1}{h(\zeta)} \phi \mathcal{M} \pi^{(L)} A_{2}^{\prime} h(\zeta) \phi^{\prime}\left(1-\pi^{(L)}\right) \psi \\
\quad+\frac{1}{h(\zeta)} \phi \mathcal{M} \pi^{(L)} A_{2}^{\prime} \phi^{\prime}\left[\psi\left(1-\pi^{(L)}\right) \psi, h(\zeta) \phi^{\prime}\right] \psi+E^{\left(1^{-}\right)}
\end{gathered}
$$

Actually, here we have used the fact that:

$$
\phi \mathcal{M} \pi^{(L)} A_{2}^{\prime}\left(1-\pi^{(L)}\right) \equiv \phi \mathcal{M} \pi^{(L)} A_{2}^{\prime} \psi\left(1-\pi^{(L)}\right)
$$

which we will also have for our specific choice of $\mathcal{M}$ (see Section 2.2 and Section 3.2 .2 ). Since $\mathcal{M} \pi^{(L)} A_{2}^{\prime}$ is smoothing of order 0 , and

$$
\left[\psi\left(1-\pi^{(L)}\right) \psi, h(\zeta) \phi^{\prime}\right]
$$

is smoothing of order 1 (by Proposition 2.11), the third term in Equation (3.3) may be taken as part of our error term. It follows, if we set

$$
T^{(0)}=\frac{1}{h(\zeta)} \phi \mathcal{M} \pi^{(L)} A_{2}^{\prime} h(\zeta) \phi^{\prime}
$$

(which is smoothing of order 0 since $\mathcal{M} \pi^{(L)} A_{2}^{\prime}$ is), we have:

$$
\frac{1}{h(\zeta)} \phi \mathcal{M} \pi^{(L)} \phi^{\prime} \mathcal{A}=\phi \pi^{(L)} \phi^{\prime}+T^{(0)}\left(1-\pi^{(L)}\right) \psi+E^{\left(1^{-}\right)}
$$

We recall the operator $U^{(2)}$ from Theorem 3.3 (we pick $\phi \prec \phi^{\prime}$ in that theorem to be $\psi \prec \psi^{\prime}$ here). Hence,

$$
\begin{aligned}
& \left(\frac{1}{h(\zeta)} \phi \mathcal{M} \pi^{(L)} \phi^{\prime}-T^{(0)} U^{(2)}\right) \mathcal{A} \\
& \quad=\phi \pi^{(L)} \phi^{\prime}+T^{(0)}\left(1-\pi^{(L)}\right) \psi-T^{(0)}\left(1-\pi^{(L)}\right) \psi^{\prime}+E^{\left(1^{-}\right)} \\
& \quad=\phi \pi^{(L)} \phi^{\prime}+E^{\left(1^{-}\right)}
\end{aligned}
$$

where, in the last line, we have used the pseudolocality of everything involved to see that $T^{(0)}\left(1-\pi^{(L)}\right) \psi \equiv T^{(0)}\left(1-\pi^{(L)}\right) \psi^{\prime}$. 
3.2.1. Formally Calculating $\mathcal{M}$. In this section, we wish to formally calculate $\mathcal{M}$. We will later show that these formal calculations can be made rigorous. Recall, we wish to have $\mathcal{M}$ such that $\mathcal{M} \pi^{(L)} A_{2}^{\prime} \pi^{(L)}=\pi^{(L)}$. We first do our calculations for the example case when we replace $A_{2}^{\prime}$ by $|z|^{4}$. This case contains essentially all of the ideas of the more complicated case for $A_{2}^{\prime}$, and will offer the reader a road map for the more complicated book keeping that will arise later.

$\pi^{(L)}$ satisfies the equation $\bar{Z}_{L} \pi^{(L)}=0$. That is, $\left(\partial_{\bar{z}}+z D_{t}\right) \pi^{(L)}=0$, and therefore,

$$
z D_{t} \pi^{(L)}=-\partial_{\bar{z}} \pi^{(L)}=-\frac{1}{2}\left(\partial_{\bar{z}}-z D_{t}\right) \pi^{(L)}=-\frac{1}{2} \bar{Z}_{R} \pi^{(L)}
$$

and the adjoint result is:

$$
\pi^{(L)} \bar{z} D_{t}=\frac{1}{2} Z_{R} \pi^{(L)}
$$

Here we have used that $Z_{R}$ and $\pi^{(L)}$ commute, since left invariant convolution operators commute with right invariant differential operators. Thus, we compute:

$$
\begin{aligned}
D_{t}^{4} \pi^{(L)}|z|^{4} \pi^{(L)} & =D_{t}^{2} \pi^{(L)} \bar{z} D_{t}|z|^{2} z D_{t} \pi^{(L)} \\
& =-\frac{1}{4} Z_{R} D_{t}^{2} \pi^{(L)}|z|^{2} \pi^{(L)} \bar{Z}_{R} \\
& =-\frac{1}{4} Z_{R} \pi^{(L)} \bar{z} D_{t} z D_{t} \pi^{(L)} \bar{Z}_{R} \\
& =\frac{1}{16} Z_{R}^{2} \pi^{(L)} \bar{Z}_{R}^{2} \\
& =\frac{1}{16} Z_{R}^{2} \bar{Z}_{R}^{2} \pi^{(L)}
\end{aligned}
$$

This leads us to the calculation:

$$
\begin{aligned}
Z_{R}^{d} \bar{Z}_{R}^{d} & =Z_{R}^{d-1} \bar{Z}_{R}^{d-1} Z_{R} \bar{Z}_{R}+(d-1) Z_{R}^{d-1} \bar{Z}_{R}^{d-1}\left[Z_{R}, \bar{Z}_{R}\right] \\
& =Z_{R}^{d-1} \bar{Z}_{R}^{d-1}\left(Z_{R} \bar{Z}_{R}+(d-1)\left[Z_{R}, \bar{Z}_{R}\right]\right) \\
& =-Z_{R}^{d-1} \bar{Z}_{R}^{d-1} \mathcal{L}_{2 d-1}^{(R)} \\
& =\cdots \\
& =(-1)^{d} \prod_{j=1}^{d} \mathcal{L}_{2 j-1}^{(R)}
\end{aligned}
$$

Combining Equations 3.5 and 3.6, we see (at least formally):

$$
D_{t}^{4} \pi^{(L)}|z|^{4} \pi^{(L)}=\frac{1}{16} \mathcal{L}_{1}^{(R)} \mathcal{L}_{3}^{(R)} \pi^{(L)}
$$

We therefore define $\mathcal{M}_{0}=16\left(D_{t} P_{3}^{(R)}\right)\left(D_{t} P_{1}^{(R)}\right) D_{t}^{2}$. This operator makes sense, since $D_{t} P_{1}^{(R)}$ and $D_{t} P_{3}^{(R)}$ are in $\mathrm{Op}_{R}\left(\mathcal{O}_{0}\right)$ and so compose well. Using that $D_{t}$ 
commutes with everything in sight, we see that (formally)

$$
\begin{aligned}
\mathcal{M}_{0} \pi^{(L)}|z|^{4} \pi^{(L)} & =16 P_{1}^{(R)} P_{3}^{(R)} D_{t}^{4} \pi^{(L)}|z|^{4} \pi^{(L)} \\
& =P_{3}^{(R)} P_{1}^{(R)} \mathcal{L}_{1}^{(R)} \mathcal{L}_{3}^{(R)} \pi^{(L)} \\
& =P_{3}^{(R)}\left(1-\pi_{1}^{(R)}\right) \mathcal{L}_{3}^{(R)} \pi^{(L)} \\
& =P_{3}^{(R)} \mathcal{L}_{3}^{(R)} \pi^{(L)} \\
& =\left(1-\pi_{3}^{(R)}\right) \pi^{(L)} \\
& =\pi^{(L)}
\end{aligned}
$$

Here we have used that $\left[\mathcal{L}_{3}^{(R)}, \pi_{1}^{(R)}\right]=0$ and that $\pi_{n}^{(R)} \pi^{(L)}=0$ for all odd $n>0$ (see Section 2.3).

We now turn to formally computing $\mathcal{M}$ in the case for $A_{2}^{\prime}$ with $b>0$. By a computation completely analogous to the one above,

$$
\begin{aligned}
& D_{t}^{a+b-1} Z_{R}^{a-b} \pi^{(L)} A_{2}^{\prime} \pi^{(L)} \\
& \quad=Z_{R}^{a-b} D_{t}^{a+b-1} \pi^{(L)} \bar{Z}_{L} z^{a} \bar{z}^{b} Z_{L} \pi^{(L)} \\
& \quad=Z_{R}^{a-b} D_{t}^{a+b-1} \pi^{(L)} z^{a} \bar{z}^{b} \bar{Z}_{L} Z_{L} \pi^{(L)}+b Z_{R}^{a-b} D_{t}^{a+b-1} \pi^{(L)} z^{a} \bar{z}^{b-1} Z_{L} \pi^{(L)} \\
& \quad=Z_{R}^{a-b} D_{t}^{a+b-1} \pi^{(L)} z^{a} \bar{z}^{b}\left[\bar{Z}_{L}, Z_{L}\right] \pi^{(L)}-a b Z_{R}^{a-b} D_{t}^{a+b-1} \pi^{(L)} z^{a-1} \bar{z}^{b-1} \pi^{(L)} \\
& \quad=\cdots \\
& \quad=2^{1-a-b}(-1)^{a+1} Z_{R}^{a} \bar{Z}_{R}^{a} \pi^{(L)}-a b D_{t} Z_{R}^{a-1} \bar{Z}_{R}^{a-1} 2^{2-a-b}(-1)^{a-1} \pi^{(L)} \\
& \quad=-2^{1-a-b} \mathcal{L}_{2(a+a b)-1}^{(R)} \prod_{j=1}^{a-1} \mathcal{L}_{2 j-1}^{(R)} \pi^{(L)}
\end{aligned}
$$

where the $\cdots$ denotes that from that point on, it follows as in the computation for $|z|^{4}$. Hence, if we define

$$
\mathcal{M}=-2^{a+b-1}\left(D_{t} P_{2(a+a b)-1}^{(R)}\right) \prod_{j=1}^{a-1}\left(D_{t} P_{2 j-1}^{(R)}\right) D_{t}^{b-1} Z_{R}^{a-b}
$$

we see that (formally),

$$
\mathcal{M} \pi^{(L)} A_{2}^{\prime} \pi^{(L)}=\pi^{(L)}
$$

Here, as before $\mathcal{M}$ makes sense, since $D_{t} P_{2 j-1}^{(R)} \in \mathrm{Op}_{R}\left(\mathcal{O}_{0}\right)$ and so we may compose as many as we like. A similar computation shows that, when $b=0$,

$$
\begin{aligned}
D_{t}^{a-1} Z_{R}^{a} \pi^{(L)} A_{2}^{\prime} \pi^{(L)} & =(-1)^{a-1} 2^{1-a} Z_{R}^{a} \bar{Z}_{R}^{a} \pi^{(L)} \\
& =-2^{1-a} \prod_{j=1}^{a} \mathcal{L}_{2 j-1}^{(R)} \pi^{(L)}
\end{aligned}
$$

and in this case, we set

$$
\mathcal{M}=-2^{a-1}\left(\prod_{j=1}^{a-1}\left(D_{t} P_{2 j-1}^{(R)}\right)\right) P_{2 a-1}^{(R)} Z_{R}^{a}
$$


In either case,

$$
\mathcal{M}= \begin{cases}D_{t}^{\frac{a+b}{2}-1}\left(D_{t} R^{(2)}\right) & \text { if } a+b \text { is even } \\ D_{t}^{\frac{a+b-1}{2}} R^{(1)} & \text { if } a+b \text { is odd }\end{cases}
$$

Note that, by the results in Section 2.2, the composition $\mathcal{M} \pi^{(L)}$ makes sense.

Remark 3.8. We now see why we needed the assumption $a \geq b$ in our operator. When $b>a$, it may be that $\pi^{(L)} A_{2}^{\prime} \pi^{(L)}$ has no inverse on $\pi^{(\bar{L})}$. This exemplified by $D_{t} \pi^{(L)} \bar{z} \pi^{(L)}=c \bar{Z}_{R} \pi^{(L)}$, which has kernel in $\pi^{(L)} L^{2}$.

3.2.2. Rigorous Calculations. The reason the calculations in Section 3.2.1 may not be rigorous is that the operator

$$
\pi^{(L)} A_{2}^{\prime} \pi^{(L)}
$$

may not make sense. To see this more simply, consider $\pi^{(L)}|z|^{2 M} \pi^{(L)}$. The Schwartz kernel of $|z|^{2 M} \pi^{(L)}$ grows as $|z| \rightarrow \infty$ (for $M$ sufficiently large) and it is therefore difficult to make sense of $\pi^{(L)}|z|^{2 M} \pi^{(L)}$. In this section, we will discuss two ways around this problem. The first method is quite simple, and involves merely localizing all of our calculations so that no composition is hard to make sense of. The second method is a bit more complicated, but will imply (for instance) that $\mathcal{M} \pi^{(L)} A_{2}^{\prime}$ is smoothing of order 0 , and so will be necessary for our purposes.

Remark 3.9. This was no problem in [Str06], as in that case $Q$ (which is the analog of our $\pi^{(L)}$ ) had a kernel which vanished rapidly for $x$ large, and therefore $Q x^{2 k} Q$ was easy to make sense of.

\section{The First Method}

The first method involves replacing the result

$$
\mathcal{M} \pi^{(L)} A_{2}^{\prime} \pi^{(L)}
$$

with a local one. That is, let $\phi \prec \phi^{\prime}$ be nested cut off functions. We will show:

$$
\phi \mathcal{M} \pi^{(L)} \phi^{\prime} A_{2}^{\prime} \pi^{(L)} \equiv \phi \pi^{(L)}
$$

Using the results in Section 2.2, we see (computing now just in the case $b>0$, the case $b=0$ is similar):

$$
\begin{aligned}
\phi \mathcal{M} \pi^{(L)} \phi^{\prime} \equiv \phi & 2^{a+b-1}\left(D_{t} P_{2(a+a b)-1}^{(R)}\right) \phi^{\prime} \\
& \times \prod_{j=1}^{a-1}\left(D_{t} P_{2 j-1}^{(R)} \phi^{\prime}\right) D_{t}^{b-1} Z_{R}^{a-b} \phi^{\prime} \pi^{(L)} \phi^{\prime}
\end{aligned}
$$

Applying the RHS of Equation (3.10) to $A_{2} \pi^{(L)}$, we may now repeat the formal argument in Section 3.2.1, which is now rigorous. The only difference is that as we move differential operators around, they sometimes land on a $\phi^{\prime}$-such terms are infinitely smoothing as all the operators involved are pseudolocal and $\phi \prec \phi^{\prime}$. It follows that:

$$
\phi \mathcal{M} \pi^{(L)} \phi^{\prime} A_{2}^{\prime} \pi^{(L)} \equiv \phi \pi^{(L)}
$$

\section{The Second Method}


The problem we are facing in this section is that it is difficult to make sense of $\pi^{(L)} A_{2}^{\prime} \pi^{(L)}$. The idea for this method is that, while we may have trouble making sense of $\pi^{(L)} A_{2}^{\prime} \pi^{(L)}$, we will be able to make sense of:

$$
\left(\left(\mathcal{M} \pi^{(L)}\right) A_{2}^{\prime}\right) \pi^{(L)}
$$

where the parentheses denote the order in which we take the composition. As usual, $R^{(j)}$ will denote an arbitrary element of $\mathrm{Op}_{R}\left(\mathcal{O}_{j}\right)$, and similarly $L^{(j)}$ will denote an arbitrary element of $\mathrm{Op}_{L}\left(\mathcal{O}_{j}\right)$. Also, we will write $R_{(j)}$ for any finite linear combination of terms of the form $\nabla_{R}^{J}$ with $|J|=j$ (recall $\nabla_{R}=\left(X_{R}, Y_{R}\right)$ ).

We have already made sense of $\mathcal{M} \pi^{(L)}$ on distributions of compact support (see the end of Section 3.2.1), and therefore we have made sense of $\mathcal{M} \pi^{(L)} A_{2}^{\prime}$ on distributions of compact support. We will show that $\mathcal{M} \pi^{(L)} A_{2}^{\prime}$ is a sum of terms of the form $R^{(0)} L^{(0)}, L^{(0)}$, and $D_{t} R^{(1)} L^{(1)}$. This will prove two things. First, it will make sense to compose $\mathcal{M} \pi^{(L)} A_{2}^{\prime}$ with $\pi^{(L)}$ (by the considerations of Section 2.2). More importantly, we will see in the proof that the $L^{(0)} \mathrm{s}$ and $R^{(0)} \mathrm{s}$ above are of the form $D_{t} L^{(2)}$ and $D_{t} R^{(2)}$ respectively, and therefore $\mathcal{M} \pi^{(L)} A_{2}^{\prime}$ is smoothing of order 0 by Remark 2.22 (and by Propositions 2.18 and 2.19). The reason this part is more important is that we could use the first method of this section to understand the compositions, but we need these considerations to understand the regularity properties of $\mathcal{M} \pi^{(L)} A_{2}^{\prime}$.

Proposition 3.10. $\mathcal{M} \pi^{(L)} A_{2}^{\prime}$ can be written as a sum of terms of the form

$$
D_{t} R^{(1)} \pi^{(L)} L^{(1)},\left(D_{t} R^{(2)}\right) \pi^{(L)}\left(D_{t} L^{(2)}\right) \text {, and } \pi^{(L)}\left(D_{t} L^{(2)}\right) \text {. }
$$

To prove Proposition 3.10 we note (in either the case $b=0$ or $b>0$ ) that

$$
\mathcal{M}=R^{(2)} \prod_{j=1}^{a-1}\left(D_{t} R^{(2)}\right) D_{t}^{b} R_{(a-b)}
$$

We will write $\mathcal{M}(a, b)$ for an operator that is a sum of terms of that form. We will be inducting on $a, b, a \geq b$. So consider, for $a \geq b, a>0$,

$$
\begin{aligned}
\mathcal{M}(a+1, b) & =R^{(2)} \prod_{j=1}^{a}\left(D_{t} R^{(2)}\right) D_{t}^{b} R_{(a+1-b)} \\
& =\left(D_{t} R^{(1)}\right) R^{(2)} \prod_{j=1}^{a-1}\left(D_{t} R^{(2)}\right) D_{t}^{b} R_{(a-b)} \\
& =\left(D_{t} R^{(1)}\right) \mathcal{M}(a, b)
\end{aligned}
$$

(In the second to last equality above, we have used that the vector space spanned by $X_{R}$ and $Y_{R}$ commutes with $\mathrm{Op}_{R}\left(\mathcal{O}_{j}\right)$, as sets.) If $a>b$,

$$
\begin{aligned}
\mathcal{M}(a, b+1) & =R^{(2)} \prod_{j=1}^{a-1}\left(D_{t} R^{(2)}\right) D_{t}^{b+1} R_{(a-b-1)} \\
& =R^{(2)} \prod_{j=1}^{a-1}\left(D_{t} R^{(2)}\right) D_{t}^{b+1} \mathcal{J}^{(R)} \mathcal{L}_{0}^{(R)} R_{(a-b-1)} \\
& =\left(D_{t} R^{(1)}\right) \mathcal{M}(a, b)
\end{aligned}
$$


where $\mathcal{J}^{(R)} \in \mathrm{Op}_{R}\left(\mathcal{O}_{2}\right)$ is the right invariant analog of the operator from Proposition 2.6. Here, we have used:

$$
R^{(2)} R^{(1)}=R^{(2)} \mathcal{L}_{0}^{(R)} \mathcal{J}^{(R)} R^{(1)}=R^{(1)} R^{(2)}
$$

(Here, again, we mean a sum of such terms in the above.) Consider (recalling that $\left.\pi^{(L)} Z_{L}=0\right)$,

$$
\begin{aligned}
\pi^{(L)} A_{2}^{\prime} & =\pi^{(L)} \bar{Z}_{L} z^{a} \bar{z}^{b} Z_{L} \\
& =\pi^{(L)}\left(\left[\bar{Z}_{L}, Z_{L}\right] z^{a} \bar{z}^{b}-a \bar{Z}_{L} z^{a-1} \bar{z}^{b}\right) \\
& =\pi^{(L)}\left(-2 z^{a} \bar{z}^{b} D_{t}-a z^{a-1} \bar{z}^{b} \bar{Z}_{L}-a b z^{a-1} \bar{z}^{b-1}\right)
\end{aligned}
$$

We therefore separate Proposition 3.10 into the same result for each of the three terms on the RHS of (3.13) (when $b=0$, the third term is 0 ). We prove these results in slightly greater generality needed here, for use in the next section.

Lemma 3.11. $\mathcal{M}(a, b) \pi^{(L)} z^{d} \bar{z}^{e}(d+e=a+b-2)$ can be written as a sum of terms of the form $D_{t} R^{(1)} \pi^{(L)} L^{(1)}$ and $\left(D_{t} R^{(2)}\right) \pi^{(L)}\left(D_{t} L^{(2)}\right)$. Here, $a \geq b \geq 0$, $a+b \geq 2$.

Proof. First we recall from Section 2.3 that $\pi^{(L)}=D_{t} L^{(2)}$. We begin by proving the result for $a=b=1$ and $a=2, b=0$ and then induct on $a \geq b$. When $a=b=1$,

$$
\begin{aligned}
\mathcal{M}(1,1) \pi^{(L)} & =R^{(2)} D_{t} \pi^{(L)} \pi^{(L)} \\
& =\left(D_{t} R^{(2)}\right) \pi^{(L)}\left(D_{t} L^{(2)}\right)
\end{aligned}
$$

and when $a=2, b=0$,

$$
\begin{aligned}
\mathcal{M}(2,0) \pi^{(L)} & =R^{(2)}\left(D_{t} R^{(2)}\right) R_{(2)} \pi^{(L)} \\
& =\left(D_{t} R^{(2)}\right) \pi^{(L)} \pi^{(L)} \\
& =\left(D_{t} R^{(2)}\right) \pi^{(L)}\left(D_{t} L^{(2)}\right)
\end{aligned}
$$

Now suppose $a \geq b$ and the result holds for $(a, b)$. We prove the result for $(a+1, b), d+e=a+b-1$. We assume first that $d>0$.

$$
\mathcal{M}(a+1, b) \pi^{(L)} z^{d} \bar{z}^{e}=\left(D_{t} R^{(1)}\right)\left(\mathcal{M}(a, b) \pi^{(L)} z^{d-1} \bar{z}^{e}\right) z
$$

By our inductive hypothesis, $\mathcal{M}(a, b) \pi^{(L)} z^{d-1} \bar{z}^{e}$ is a sum of terms of the form

$$
D_{t} R^{(1)} \pi^{(L)} L^{(1)} \text { and }\left(D_{t} R^{(2)}\right) \pi^{(L)}\left(D_{t} L^{(2)}\right)
$$


Thus, we may replace $\mathcal{M}(a, b) \pi^{(L)} z^{d-1} \bar{z}^{e}$ in the RHS of (3.14) with a sum of such terms. But,

$$
\begin{aligned}
\left(D_{t} R^{(1)}\right) D_{t} R^{(1)} \pi^{(L)} L^{(1)} z & \\
= & \left(D_{t} R^{(2)}\right) \pi^{(L)} D_{t}\left(\left[L^{(1)}, z\right]\right)+\left(D_{t} R^{(2)}\right)\left(\pi^{(L)} D_{t}\left[\pi^{(L)}, z\right] L^{(1)}\right) \\
& +\left(D_{t} R^{(2)}\right) \pi^{(L)} z D_{t} \pi^{(L)} L^{(1)} \\
= & \left(D_{t} R^{(2)}\right) \pi^{(L)} D_{t} L^{(2)}+\left(D_{t} R^{(2)}\right) \bar{Z}_{R} \pi^{(L)} L^{(1)} \\
= & \left(D_{t} R^{(2)}\right) \pi^{(L)}\left(D_{t} L^{(2)}\right)+D_{t} R^{(1)} \pi^{(L)} L^{(1)}
\end{aligned}
$$

Note, the composition

$$
\left(D_{t} R^{(2)}\right) \pi^{(L)}\left(z D_{t} \pi^{(L)} L^{(1)}\right)
$$

actually makes sense by the equality $z D_{t} \pi^{(L)}=-\frac{1}{2} \bar{Z}_{R} \pi^{(L)}$. In a similar manner we see

$$
\begin{aligned}
\left(D_{t} R^{(1)}\right) & \left(D_{t} R^{(2)}\right) \pi^{(L)}\left(D_{t} L^{(2)}\right) z \\
& =D_{t} R^{(1)} \pi^{(L)} L^{(1)}+\left(D_{t} R^{(2)}\right) \pi^{(L)}\left(D_{t} L^{(2)}\right)
\end{aligned}
$$

If instead $d=0$ and $e>0$ the proof follows just as above, except we use the equality:

instead of the equality

$$
\pi^{(L)} \bar{z} D_{t}=\frac{1}{2} Z_{R} \pi^{(L)}
$$

$$
D_{t} z \pi^{(L)}=-\frac{1}{2} \bar{Z}_{R} \pi^{(L)}
$$

This proves the result for $(a+1, b)$.

Now we assume $a>b \geq 0$ and that we have the result for $(a, b)$. To prove the result for $(a, b+1)$, we simply use (3.12) instead of (3.11), and the proof remains the same.

Lemma 3.12. $\mathcal{M}(a, b) \pi^{(L)} z^{d} \bar{z}^{e} \bar{Z}_{L}(d+e=a+b-1)$ can be written as a sum of terms of the form $D_{t} R^{(1)} \pi^{(L)} L^{(1)}$ and $\left(D_{t} R^{(2)}\right) \pi^{(L)}\left(D_{t} L^{(2)}\right)$. Here, $a \geq b \geq 0$, $a>0$.

Proof. This follows by an induction similar to the one in Lemma 3.11.

To complete the proof of Proposition 3.10, we need to consider terms of the form $\pi^{(L)} z^{a} \bar{z}^{b} D_{t}$. These terms will require a more delicate analysis than those in the pervious lemmas, and before we embark on these difficulties, we prove a result along the lines of the previous lemmas, that is good enough for many of our purposes, and will be useful later.

Lemma 3.13. $\mathcal{M}(a, b) \pi^{(L)} z^{d} \bar{z}^{e} D_{t}(d+e=a+b)$ can be written as a sum of terms of the form $D_{t} R^{(1)} \pi^{(L)} L^{(1)}$ and $R^{(0)} \pi^{(L)} L^{(0)}$. Here, $a \geq b \geq 0$, $a>0$. Note that $R^{(0)}$ is not necessarily of the form $D_{t} R^{(2)}$.

Proof. This follows by an induction similar to the one in Lemma 3.11. 
We now return to the more delicate analysis of terms of the form $\pi^{(L)} z^{a} \bar{z}^{b} D_{t}$. Consider,

$$
\begin{aligned}
\mathcal{M} \pi^{(L)} z^{a} \bar{z}^{b} D_{t} & =-2^{a+b-1} P_{2(a+a b)-1}^{(R)} \prod_{j=1}^{a-1}\left(D_{t} P_{2 j-1}^{(R)}\right) Z_{R}^{a-b} \pi^{(L)} D_{t}^{b} \bar{z}^{b} z^{a} D_{t} \\
& =-2^{a-1} P_{2(a+a b)-1}^{(R)} \prod_{j=1}^{a-1}\left(D_{t} P_{2 j-1}^{(R)}\right) Z_{R}^{a} \pi^{(L)} z^{a} D_{t}
\end{aligned}
$$

Thus, if we define $\mathcal{M}(a)$ to be any sum of terms of the form:

$$
\left(1+D_{t} R^{(2)}\right) P_{2 n_{a}-1}^{(R)} \prod_{j=1}^{a-1}\left(D_{t} P_{2 n_{j}-1}^{(R)}\right) Z_{R}^{a}
$$

where all the $n_{j}>0$. Then to complete the proof of Proposition 3.10 it suffices to prove the result:

Lemma 3.14. $\mathcal{M}(a) \pi^{(L)} z^{a} D_{t}$ can be written as a sum of terms of the form

$$
D_{t} R^{(1)} \pi^{(L)} L^{(1)},\left(D_{t} R^{(2)}\right) \pi^{(L)}\left(D_{t} L^{(2)}\right) \text {, and } \pi^{(L)}\left(D_{t} L^{(2)}\right) .
$$

Here, $a>0$.

Before we prove Lemma 3.14 we need an intermediary lemma:

Lemma 3.15. $\mathcal{M}(a, 0) \pi^{(L)} L^{(1)} z^{(a-1)} D_{t}$ can be written as a sum of terms of the form $D_{t} R^{(1)} \pi^{(L)} L^{(1)}$ and $\left(D_{t} R^{(2)}\right) \pi^{(L)}\left(D_{t} L^{(2)}\right)$. Here, $a>0$.

Proof. This follows by an induction similar to the one in Lemma 3.11.

Proof of Lemma 3.14. We induct on $a$. Our base case is $a=1$ :

$$
\begin{aligned}
\mathcal{M}(1) \pi^{(L)} z D_{t}= & \left(1+D_{t} R^{(2)}\right) P_{2 n-1}^{(R)} Z_{R} \pi^{(L)} z D_{t} \\
= & \left(1+D_{t} R^{(2)}\right) P_{2 n-1}^{(R)} Z_{R} \pi^{(L)}\left[\pi^{(L)}, z\right] D_{t} \\
& +\left(1+D_{t} R^{(2)}\right) P_{2 n-1}^{(R)} Z_{R} \bar{Z}_{R} \pi^{(L)}
\end{aligned}
$$

Now the first term in the RHS of the above equation is of the desired form by Lemma 3.15. We consider now the second:

$$
\begin{aligned}
\left(1+D_{t} R^{(2)}\right) P_{2 n-1}^{(R)} Z_{R} \bar{Z}_{R} \pi^{(L)} & =\left(1+D_{t} R^{(2)}\right) P_{2 n-1}^{(R)}\left(\mathcal{L}_{2 n-1}^{(R)}+C D_{t}\right) \pi^{(L)} \\
& =\left(1+D_{t} R^{(2)}\right)\left(1-\pi_{2 n-1}^{(R)}\right) \pi^{(L)}+D_{t} R^{(2)} \pi^{(L)}
\end{aligned}
$$

This completes the result for $a=1$ by using the fact that $\pi_{2 n-1}^{(R)}=D_{t} R^{(2)}$ and $\pi^{(L)}=D_{t} L^{(2)}$.

We assume we have the result for $a$ and prove it for $a+1$.

$$
\begin{aligned}
\mathcal{M}(a+1) \pi^{(L)} z^{a+1} D_{t}= & \mathcal{M}(a+1) \pi^{(L)} z D_{t} \pi^{(L)} z^{a} \\
& +\mathcal{M}(a+1) \pi^{(L)}\left[\pi^{(L)}, z\right] z^{a} D_{t}
\end{aligned}
$$


The second term on the RHS of the above equation is of the desired form by Lemma 3.15. We therefore consider just the first:

$$
\begin{aligned}
\mathcal{M}(a+1) & \pi^{(L)} z D_{t} \pi^{(L)} z^{a}=\mathcal{M}(a+1) \bar{Z}_{R} \pi^{(L)} z^{a} \\
= & \left(1+D_{t} R^{(2)}\right) P_{2 n_{a+1}-1}^{(R)} \prod_{j=1}^{a}\left(D_{t} P_{2 n_{j}-1}^{(R)}\right) Z_{R}^{a+1} \bar{Z}_{R} \pi^{(L)} z^{a} \\
= & \left(1+D_{t} R^{(2)}\right) P_{2 n_{a+1}-1}^{(R)} \prod_{j=1}^{a}\left(D_{t} P_{2 n_{j}-1}^{(R)}\right) Z_{R} \bar{Z}_{R} Z_{R}^{a} \pi^{(L)} z^{a} \\
& +C\left(1+D_{t} R^{(2)}\right) P_{2 n_{a+1}-1}^{(R)} \prod_{j=1}^{a}\left(D_{t} P_{2 n_{j}-1}^{(R)}\right) D_{t} Z_{R}^{a} \pi^{(L)} z^{a}
\end{aligned}
$$

Now the second term on the RHS of the above equation is of the desired form by our inductive hypothesis, so we consider only the first:

$$
\begin{aligned}
\left(1+D_{t} R^{(2)}\right) P_{2 n_{a+1}-1}^{(R)} \prod_{j=1}^{a}\left(D_{t} P_{2 n_{j}-1}^{(R)}\right) Z_{R} \bar{Z}_{R} Z_{R}^{a} \pi^{(L)} z^{a} \\
=C_{1}\left(1+D_{t} R^{(2)}\right) P_{2 n_{a+1}-1}^{(R)} \prod_{j=1}^{a}\left(D_{t} P_{2 n_{j}-1}^{(R)}\right) \mathcal{L}_{2 n_{a+1}-1}^{(R)} Z_{R}^{a} \pi^{(L)} z^{a} \\
\\
\quad+C_{2}\left(1+D_{t} R^{(2)}\right) P_{2 n_{a+1}-1}^{(R)} \prod_{j=1}^{a}\left(D_{t} P_{2 n_{j}-1}^{(R)}\right) D_{t} Z_{R}^{a} \pi^{(L)} z^{a}
\end{aligned}
$$

The second term on the RHS of the above equation is of the desired form by our inductive hypothesis, so we consider only the first:

$$
\begin{aligned}
(1+ & \left.D_{t} R^{(2)}\right) P_{2 n_{a+1}-1}^{(R)} \prod_{j=1}^{a}\left(D_{t} P_{2 n_{j}-1}^{(R)}\right) \mathcal{L}_{2 n_{a+1}-1}^{(R)} Z_{R}^{a} \pi^{(L)} z^{a} \\
& =\left(1+D_{t} R^{(2)}\right) P_{2 n_{a+1}-1}^{(R)} \mathcal{L}_{2 n_{a+1}-1}^{(R)} \prod_{j=1}^{a}\left(D_{t} P_{2 n_{j}-1}^{(R)}\right) Z_{R}^{a} \pi^{(L)} z^{a} \\
& =\left(1+D_{t} R^{(2)}\right)\left(1-\pi_{2 n_{a+1}-1}^{(R)}\right) \prod_{j=1}^{a}\left(D_{t} P_{2 n_{j}-1}^{(R)}\right) Z_{R}^{a} \pi^{(L)} z^{a} \\
& =\left(1+D_{t} R^{(2)}\right)\left(1+D_{t} R^{(2)}\right) P_{2 n_{a}-1}^{(R)} \prod_{j=1}^{a-1}\left(D_{t} P_{2 n_{j}-1}^{(R)}\right) Z_{R}^{a} \pi^{(L)} z^{a} D_{t}
\end{aligned}
$$

which is of the desired form by our inductive hypothesis.

Combining Lemma 3.14 with the remarks preceding it, we get:

Lemma 3.16. $\mathcal{M} \pi^{(L)} z^{a} \bar{z}^{b} D_{t}$ can be written as a sum of terms of the form

$$
D_{t} R^{(1)} \pi^{(L)} L^{(1)},\left(D_{t} R^{(2)}\right) \pi^{(L)}\left(D_{t} L^{(2)}\right) \text {, and } \pi^{(L)}\left(D_{t} L^{(2)}\right) \text {. }
$$

Here, $a>0$.

which completes the proof of Proposition 3.10.

Proposition 3.17. $\mathcal{M} \pi^{(L)} A_{2}^{\prime} \pi^{(L)} \equiv \pi^{(L)}$ 
Proof. Let $\psi_{1} \prec \psi_{2} \in C_{0}^{\infty}$. Then the above calculations (along with the results of Section 2.2) show that

$$
\psi_{1} \mathcal{M} \pi^{(L)} \psi_{2} A_{2}^{\prime} \pi^{(L)} \equiv \psi_{1} \mathcal{M} \pi^{(L)} A_{2}^{\prime} \pi^{(L)}
$$

Thus by our computations in the first method, we see that:

$$
\psi_{1} \mathcal{M} \pi^{(L)} A_{2}^{\prime} \pi^{(L)} \equiv \psi_{1} \pi^{(L)}
$$

completing the claim.

Remark 3.18. Actually,

$$
\mathcal{M} \pi^{(L)} A_{2}^{\prime} \pi^{(L)}=\pi^{(L)}
$$

This follows from a more delicate computation than the one above, though essentially the same. We will not need this stronger result, and so will not bother proving it here.

3.2.3. Error Terms. In this section, we wish to show that $\mathcal{M} \pi^{(L)} A_{3}, \mathcal{M} \pi^{(L)} A_{4}$, and $\mathcal{M} \pi^{(L)} A_{5}$ are smoothing of order $1^{-}$. Actually, $\mathcal{M} \pi^{(L)} A_{3}=0$, so we are really only concerned with $A_{4}$ and $A_{5}$. First we will need a lemma.

Lemma 3.19. The equations

$$
\begin{gathered}
\left(D_{t} R^{(2)}\right) \pi^{(L)} L^{(0)} z,\left(D_{t} R^{(2)}\right) \pi^{(L)} L^{(0)} \bar{z} \\
D_{t} R^{(1)} \pi^{(L)} L^{(1)} z, \text { and } D_{t} R^{(1)} \pi^{(L)} L^{(1)} \bar{z}
\end{gathered}
$$

can each be written as a sum of terms of the form $R^{(1)} \pi^{(L)} L^{(1)}, R^{(0)} \pi^{(L)} L^{(1)}$, and $R^{(1)} \pi^{(L)} L^{(0)}$ and are therefore smoothing of order $1^{-}$.

Proof. Consider,

$$
\begin{aligned}
\left(D_{t} R^{(2)}\right) \pi^{(L)} L^{(0)} z & =R^{(2)} \pi^{(L)} z D_{t} \pi^{(L)} L^{(0)}+\left(D_{t} R^{(2)}\right) \pi^{(L)} L^{(1)} \\
& =R^{(2)} \bar{Z}_{R} \pi^{(L)} L^{(0)}+\left(D_{t} R^{(2)}\right) \pi^{(L)} L^{(1)} \\
& =R^{(1)} \pi^{(L)} L^{(0)}+\left(D_{t} R^{(2)}\right) \pi^{(L)} L^{(1)}
\end{aligned}
$$

A similar proof yields the result for $\left(D_{t} R^{(2)}\right) \pi^{(L)} L^{(0)} \bar{z}$.

Turning our attention to $D_{t} R^{(1)} \pi^{(L)} L^{(1)} z$, we see

$$
\begin{aligned}
D_{t} R^{(1)} \pi^{(L)} L^{(1)} z & =R^{(1)} \pi^{(L)} z D_{t} \pi^{(L)} L^{(1)}+R^{(1)} \pi^{(L)} D_{t} L^{(2)} \\
& =R^{(1)} Z_{R} \pi^{(L)} L^{(1)}+R^{(1)} \pi^{(L)} D_{t} L^{(2)} \\
& =R^{(0)} \pi^{(L)} L^{(1)}+R^{(1)} \pi^{(L)} D_{t} L^{(2)}
\end{aligned}
$$

and a similar proof yields the result for $D_{t} R^{(1)} \pi^{(L)} L^{(1)} \bar{z}$. The fact that these operators are smoothing of order $1^{-}$can be found in Section 2.4.

Proposition 3.20. $\mathcal{M} \pi^{(L)} A_{5}$ is smoothing of order $1^{-}$.

Proof. It suffices to prove the result for

$$
\mathcal{M} \pi^{(L)} z^{d} \bar{z}^{e}
$$

where $d+e=a+b-1$. If $(a, b) \neq(1,0)$, follows from Lemma 3.19 combined with Lemma 3.11. If $(a, b)=(1,0)$ this lemma is obvious, as $\mathcal{M} \pi^{(L)}=R^{(1)} \pi^{(L)}$ is smoothing of order 1 by Corollary 2.20. 
Proposition 3.21. $\mathcal{M} \pi^{(L)} A_{4}$ is smoothing of order $1^{-}$.

Proof. We consider a term of the form:

$$
\mathcal{M} \pi^{(L)} z^{d} \bar{z}^{e} g(z, \bar{z}, t) \bar{Z}_{L}
$$

where $g(z, \bar{z}, t) \in \mathbb{C}^{\infty}$ and $d+e=a+b$. Consider,

$$
\mathcal{M} \pi^{(L)} z^{d} \bar{z}^{e} g(z, \bar{z}, t) \bar{Z}_{L}=\mathcal{M} \pi^{(L)} z^{d} \bar{z}^{e} \bar{Z}_{L} g(z, \bar{z}, t)-\mathcal{M} \pi^{(L)} z^{d} \bar{z}^{e}\left(\bar{Z}_{L} g\right)(z, \bar{z}, t)
$$

The second term on the RHS is taken care of the form covered in Proposition 3.20. Considering just the first, if $d>0$, we see:

$$
\mathcal{M} \pi^{(L)} z^{d} \bar{z}^{e} \bar{Z}_{L}=\mathcal{M} \pi^{(L)} z^{d-1} \bar{z}^{e} \bar{Z}_{L} z
$$

which is smoothing of order $1^{-}$by combining Lemmas 3.19 and 3.12. If $d=0$, we see

$$
\mathcal{M} \pi^{(L)} \bar{z}^{a+b} \bar{Z}_{L}=\mathcal{M} \pi^{(L)} \bar{z}^{a+b-1} \bar{Z}_{L} \bar{z}-(a+b) \mathcal{M} \pi^{(L)} \bar{z}^{a+b-1}
$$

The second term on the RHS of the above equation is of the form covered in Proposition 3.20. The first term can be seen to be smoothing or order $1^{-}$by combining Lemmas 3.19 and 3.12 .

3.3. Properties of the Parametrix. The results of Section 3.2.2 and Section 3.2 .3 are all that we required for the computations leading up to Equation (3.4). Thus those considerations complete the construction of the parametrix. That is, if we set:

We see that

$$
J=\frac{1}{h(\zeta)} \phi \mathcal{M} \pi^{(L)} \phi^{\prime}-T^{(0)} U^{(2)}+\phi U^{(2)}
$$

$$
J \mathcal{A}=\phi+E^{\left(1^{-}\right)}
$$

where $E^{\left(1^{-}\right)}$is smoothing of order $1^{-}$. Recall that $T^{(0)}=\phi \frac{1}{h(\zeta)} \mathcal{M} \pi^{(L)} A_{2}^{\prime} h(\zeta) \phi^{\prime}$ and therefore was smoothing of order 0 . From this, it follows that $J\left(1-\pi^{(L)}\right)$ is smoothing of order 2. Of course, we may iterate Equation (3.15), in the usual way, to get (for each $N \in \mathbb{N}$ ) a parametrix $J_{N}$ such that:

$$
J_{N} \mathcal{A}=\phi+E^{(N)}
$$

where $E^{(N)}$ is smoothing of order $N$.

By Equation (3.8) we see that:

$$
\begin{aligned}
\mathcal{M} \pi^{(L)} & = \begin{cases}D_{t}^{\frac{a+b}{2}-1}\left(D_{t} R^{(2)}\right) \pi^{(L)} & \text { if } a+b \text { is even, } \\
D_{t}^{\frac{a+b-1}{2}} R^{(1)} \pi^{(L)} & \text { if } a+b \text { is odd }\end{cases} \\
& = \begin{cases}V^{(0)} D_{t}^{\frac{a+b}{2}-1} \pi^{(L)} & \text { if } a+b \text { is even, } \\
V^{(1)} D_{t}^{\frac{a+b-1}{2}} \pi^{(L)} & \text { if } a+b \text { is odd }\end{cases}
\end{aligned}
$$

where $V^{(0)}$ is smoothing of order 0 , and $V^{(1)}$ is smoothing of order 1 . (To see $R^{(1)} \pi^{(L)}$ is smoothing of order 1, we have used Corollary 2.20.) From here, the regularity properties of $\mathcal{A}$ follow easily. We state these in terms of inequalities. In the propositions below, $p$ is some fixed real number, $1<p<\infty$. 
Proposition 3.22. For $1<p<\infty$, $\mathcal{A}$ loses at most $\frac{a+b}{2}-1$ derivatives near 0 in $L^{p}$ Sobolev spaces; that is, if $\phi \prec \phi^{\prime} \prec \phi^{\prime \prime}$ are cut off functions supported sufficiently close to $0 \in \mathbb{H}^{1}$ and $u$ is a distribution, we have (for every $s \in \mathbb{R}$ )

$$
\|\phi u\|_{L_{s}^{p}} \lesssim\left\|\phi^{\prime \prime} \mathcal{A} u\right\|_{L_{s+\frac{a+b}{2}-1}^{p}}+\left\|\phi^{\prime \prime} u\right\|_{-\infty}
$$

In fact, if $a+b$ is even,

$$
\begin{aligned}
\|\phi u\|_{L_{s}^{p}} & \lesssim \sum_{|I| \leq 2}\left\|\nabla^{I} \phi u\right\|_{L_{s-1}^{p}} \\
& \lesssim\left\|\phi^{\prime} D_{t}^{\frac{a+b}{2}} \pi^{(L)} \phi^{\prime \prime} \mathcal{A} u\right\|_{L_{s-1}^{p}}+\left\|\phi^{\prime \prime} \mathcal{A} u\right\|_{L_{s-1}^{p}}+\left\|\phi^{\prime \prime} u\right\|_{-\infty}
\end{aligned}
$$

Or, if $a+b$ is odd,

$$
\begin{aligned}
\|\phi u\|_{L_{s}^{p}} & \lesssim \sum_{|I| \leq 2}\left\|\nabla^{I} \phi u\right\|_{L_{s-1}^{p}} \\
& \lesssim\left\|\phi^{\prime} D_{t}^{\frac{a+b+1}{2}} \pi^{(L)} \phi^{\prime \prime} \mathcal{A} u\right\|_{L_{s-1-\frac{1}{2}}^{p}}+\left\|\phi^{\prime \prime} \mathcal{A} u\right\|_{L_{s-1}^{p}}+\left\|\phi^{\prime \prime} u\right\|_{-\infty}
\end{aligned}
$$

This Proposition holds in the more general case when $A_{6} \neq 0$ (see Section 3.4).

Remark 3.23. Since $\pi^{(L)}$ is actually micro-supported in the + microlocalization (see Section 1.3),

$$
\left\|\phi^{\prime} D_{t}^{\frac{a+b+1}{2}} \pi^{(L)} \phi^{\prime \prime} \mathcal{A} u\right\|_{L_{s-1-\frac{1}{2}}^{p}}+\left\|\phi^{\prime \prime} u\right\|_{-\infty} \approx\left\|\phi^{\prime \prime} \pi^{(L)} \phi^{\prime \prime} \mathcal{A} u\right\|_{L_{s+\frac{a+b}{2}-1}^{p}}+\left\|\phi^{\prime \prime} u\right\|_{-\infty}
$$

and

$$
\left\|\phi^{\prime} D_{t}^{\frac{a+b}{2}} \pi^{(L)} \phi^{\prime \prime} \mathcal{A} u\right\|_{L_{s-1}^{p}}+\left\|\phi^{\prime \prime} u\right\|_{-\infty} \approx\left\|\phi^{\prime \prime} \pi^{(L)} \phi^{\prime \prime} u\right\|_{L_{s+\frac{a+b}{2}-1}^{p}}+\left\|\phi^{\prime \prime} u\right\|_{-\infty}
$$

Thereby combining both the $a+b$ is even and the $a+b$ is odd cases of Proposition 3.22 into one conclusion.

Proposition 3.24. $\mathcal{A}$ loses at most $\frac{a+b}{2}-1$ derivatives near 0 in Lipschitz spaces; that is, if $\phi \prec \phi^{\prime} \prec \phi^{\prime \prime}$ are cut off functions supported sufficiently close to $0 \in \mathbb{H}^{1}$, and $u$ is a distribution, we have (for every $\alpha>0$, with $\alpha+\frac{a+b}{2}-1>0$ ),

$$
\|\phi u\|_{\Lambda_{\alpha}} \lesssim\left\|\phi^{\prime \prime} \mathcal{A} u\right\|_{\Lambda_{\alpha+\frac{a+b}{2}-1}}+\left\|\phi^{\prime \prime} u\right\|_{-\infty}
$$

In fact, if $a+b$ is even,

$$
\|\phi u\|_{\Lambda_{\alpha}} \lesssim\left\|\phi^{\prime} D_{t}^{\frac{a+b}{2}-1} \pi^{(L)} \phi^{\prime \prime} \mathcal{A} u\right\|_{\Lambda_{\alpha}}+\left\|\phi^{\prime \prime} \mathcal{A} u\right\|_{\Lambda_{\alpha-1}}+\left\|\phi^{\prime \prime} u\right\|_{-\infty}
$$

and if $a+b$ is odd,

$$
\|\phi u\|_{\Lambda_{\alpha}} \lesssim\left\|\phi^{\prime} D_{t}^{\frac{a+b-1}{2}} \pi^{(L)} \phi^{\prime \prime} \mathcal{A} u\right\|_{\Lambda_{\alpha-\frac{1}{2}}}+\left\|\phi^{\prime \prime} \mathcal{A} u\right\|_{\Lambda_{\alpha-1}}+\left\|\phi^{\prime \prime} u\right\|_{-\infty}
$$

For results when $A_{6} \neq 0$, see Proposition 3.31.

Proposition 3.25. For $1<p<\infty$, $\mathcal{A}$ "loses at most $a+b-2$ derivatives near 0 in $N L^{p}$ Sobolev spaces;" that is, if $\phi \prec \phi^{\prime} \prec \phi^{\prime \prime}$ are cut off functions supported 
sufficiently close to $0 \in \mathbb{H}^{1}$, and $u$ is a distribution, we have (for every $k \geq 0$, with $k-a-b+2 \geq 0$ ),

$$
\|\phi u\|_{N L_{k}^{p}} \lesssim\left\|\phi^{\prime \prime} \mathcal{A} u\right\|_{N L_{k-a-b+2}^{p}}+\left\|\phi^{\prime \prime} u\right\|_{-\infty}
$$

In fact, if $a+b$ is even,

$$
\|\phi u\|_{N L_{k}^{p}} \lesssim\left\|\phi^{\prime} D_{t}^{\frac{a+b}{2}-1} \pi^{(L)} \phi^{\prime \prime} \mathcal{A} u\right\|_{N L_{k}^{p}}+\left\|\phi^{\prime \prime} \mathcal{A} u\right\|_{N L_{k-2}^{p}}+\left\|\phi^{\prime \prime} u\right\|_{-\infty}
$$

and if $a+b$ is odd,

$$
\|\phi u\|_{N L_{k}^{p}} \lesssim\left\|\phi^{\prime} D_{t}^{\frac{a+b-1}{2}} \pi^{(L)} \phi^{\prime \prime} \mathcal{A} u\right\|_{N L_{k-1}^{p}}+\left\|\phi^{\prime \prime} \mathcal{A} u\right\|_{N L_{k-2}^{p}}+\left\|\phi^{\prime \prime} u\right\|_{-\infty}
$$

This Proposition holds in the more general case when $A_{6} \neq 0$ (see Section 3.4).

3.4. When $A_{6} \neq 0$. We now turn to the case when $A_{6} \neq 0$. The first step is to see that we again have Theorem 3.3. To do this, we first need the proper analog of Proposition 3.4. Indeed, we define $\mathcal{A}_{0}=A_{1}+A_{2}+A_{6}$, so that

$$
\mathcal{A}_{0}=Z_{L} \bar{Z}_{L}+\bar{Z}_{L} F(\zeta) Z_{L}
$$

where,

$$
F(z, t)=z^{a} \bar{z}^{b} h(z, t)+\sum_{\substack{d+e=a+b+1 \\ 0 \leq d, e}} z^{d} \bar{z}^{e} g_{d, e}^{(4)}(z, t)
$$

then we have:

Proposition 3.26. Suppose $\phi$ is a cut off function supported sufficiently close to $0 \in \mathbb{H}^{1}$. Then, there exists a pseudodifferential convolution operator $S^{(2)}$, of type 2, such that

$$
S^{(2)} \mathcal{A}_{0}=F(\zeta) \phi+\kappa^{(1)}
$$

where $\kappa^{(1)}$ is a pseudodifferential convolution operator of type 1 .

Proof. This follows just as Proposition 3.4.

Theorem 3.27. Let $\phi \prec \phi^{\prime}$ be a cut off functions with support sufficiently close to $0 \in \mathbb{H}^{1}$. Then there exists a pseudodifferential convolution operator $U^{(2)}$ of type 2 , such that:

$$
U^{(2)} \mathcal{A}=\phi\left(1-\pi^{(L)}\right) \phi^{\prime}+\kappa^{(1)}
$$

where $\kappa^{(1)}$ is a pseudodifferential convolution operator of type 1 .

Proof. This follows just as Theorem 3.3.

We take $\mathcal{M}$ just as in the construction when $A_{6}=0$. Ie, we take $\mathcal{M}$ so that $\mathcal{M} \pi^{(L)} A_{2}^{\prime} \pi^{(L)}=\pi^{(L)}$. Then, if we define $T^{(0)}=\frac{1}{h(\zeta)} \phi \pi^{(L)} A_{2}^{\prime} h(\zeta) \phi^{\prime}$, which is smoothing of order 0 , we see (as in the construction of the parametrix in the case $A_{6}=0$, see Section 3.2 and Section 3.3) that if

we have:

$$
J_{1}=\frac{1}{h(\zeta)} \phi \mathcal{M} \pi^{(L)} \phi^{\prime}-T^{(0)} U^{(2)}+\phi U^{(2)}
$$

$$
J_{1} \mathcal{A}=\phi+\frac{\phi}{h} \mathcal{M} \pi^{(L)} A_{6}+E^{\left(1^{-}\right)}
$$

where $E^{\left(1^{-}\right)}$is smoothing of order $1^{-}$. 
The proof will proceed as follows. We will show that $\mathcal{M} \pi^{(L)} A_{6}$ can be written as a sum of terms of the form $V^{\left(0^{-}\right)} z, V^{\left(0^{-}\right)} \bar{z}$, and $E^{\left(1^{-}\right)}$, where $V^{\left(0^{-}\right)}$denotes an operator which is smoothing of order $0^{-}$. We can then iterate Equation (3.19) to invert $\mathcal{A}$ modulo a term that is smoothing of order $1^{-}$plus a term that vanishes (to any finite order we choose) as $z \rightarrow 0$. At this point, we satisfy ourselves with using $C^{L}$ functions in our parametrix (where $L$ is any fixed integer we chose) and complete the parametrix construction with the following proposition:

Proposition 3.28. For every $\phi \in C_{0}^{\infty}$ supported sufficiently close to $0 \in \mathbb{H}^{1}$, there exists a pseudodifferential convolution operator, $S^{(2)}$, of type 2 , such that for every $L \in \mathbb{N}$, there exists an $M$ such that for every multi-index $\alpha$ with $|\alpha|=M$, there exists a function $f_{\alpha} \in C^{L}$, with:

$$
f_{\alpha} S^{(2)} \mathcal{A}=z^{\alpha} \phi+f_{\alpha} E^{(1)}
$$

where $E^{(1)}$ is a pseudodifferential convolution operator of type 1.

Proof. Let $S^{(2)}$ be as in Proposition 3.26, and take $f_{\alpha}=\frac{z^{\alpha}}{F(\zeta)}$, which will be $C^{L}$ provided $|\alpha|$ is sufficiently large. The proof then follows immediately.

Proposition 3.29. $\mathcal{M} \pi^{(L)} A_{6}$ can be written as a sum of terms of the form

$$
\begin{aligned}
& R^{(0)}\left(D_{t} L^{(2)}\right) f(z, t) z \\
& R^{(0)}\left(D_{t} L^{(2)}\right) f(z, t) \bar{z}
\end{aligned}
$$

and terms that are smoothing of order $1^{-}$. Here, $f$ is a $C^{\infty}$ function that may change from term to term. We denote these three terms by $V_{z}^{\left(0^{-}\right)} z, V_{\bar{z}}^{\left(0^{-}\right)} \bar{z}$, and $E^{\left(1^{-}\right)}$respectively.

Proof. Consider, $\mathcal{M} \pi^{(L)} A_{6}$ is a sum of terms of the form $(d+e=a+b+1)$

$$
\begin{aligned}
\mathcal{M} \pi^{(L)} \bar{Z}_{L} z^{d} \bar{z}^{e} g(z, \bar{z}, t) Z_{L}=\mathcal{M} \pi^{(L)}\left[\bar{Z}_{L}, Z_{L}\right] z^{d} \bar{z}^{e} g(z, t) \\
-e \mathcal{M} \pi^{(L)} \bar{Z}_{L} z^{d} \bar{z}^{e-1} g(z, t)-\mathcal{M} \pi^{(L)} \bar{Z}_{L} z^{d} \bar{z}^{e}\left(Z_{L} g\right)(z, \bar{z}, t)
\end{aligned}
$$

The second and third terms are smoothing of order $1^{-}$by Propositions 3.20 and 3.21. For the first term, if $d>0$, we write:

$$
\mathcal{M} \pi^{(L)} D_{t} z^{d} \bar{z}^{e}=\mathcal{M} \pi^{(L)} z^{d-1} \bar{z}^{e} D_{t} z
$$

and Lemma 3.13 applies to see this as $V_{z}^{\left(0^{-}\right)} z+E^{\left(1^{-}\right)}$. If, instead, $d=0$ but $e>0$, then we get $V_{\bar{z}}^{\left(0^{-}\right)} \bar{z}+E^{\left(1^{-}\right)}$instead.

This shows that:

$$
J_{1} \mathcal{A}=\phi+\phi \frac{1}{h} V_{z}^{\left(0^{-}\right)} z+\phi \frac{1}{h} V_{\bar{z}}^{\left(0^{-}\right)} \bar{z}+E^{\left(1^{-}\right)}
$$

we define (with $\psi \prec \phi$ )

$$
J_{2}=\psi J_{1}-\psi \frac{1}{h} V_{z}^{\left(0^{-}\right)} z J_{1}-\psi \frac{1}{h} V_{\bar{z}}^{\left(0^{-}\right)} \bar{z} J_{1}
$$


Then,

$$
\begin{aligned}
J_{2} \mathcal{A}=\psi & +\psi \frac{1}{h} V_{z}^{\left(0^{-}\right)} z \phi \frac{1}{h} V_{z}^{\left(0^{-}\right)} z \\
& +\psi \frac{1}{h} V_{z}^{\left(0^{-}\right)} z \phi \frac{1}{h} V_{\bar{z}}^{\left(0^{-}\right)} \bar{z} \\
& +\psi \frac{1}{h} V_{\bar{z}}^{\left(0^{-}\right)} \bar{z} \phi \frac{1}{h} V_{z}^{\left(0^{-}\right)} z \\
& +\psi \frac{1}{h} V_{\bar{z}}^{\left(0^{-}\right)} \bar{z} \phi \frac{1}{h} V_{\bar{z}}^{\left(0^{-}\right)} \bar{z} \\
& +E^{\left(1^{-}\right)}
\end{aligned}
$$

We now use the fact that (as can be easily seen) $\left[z\right.$ or $\left.\bar{z}, V_{z \text { or } \bar{z}}^{\left(0^{-}\right)}\right]$is smoothing of order $1^{-}$to see:

$$
J_{2} \mathcal{A}=\psi+\psi \sum_{|I|=2}\left(\phi \frac{1}{h} V_{z}^{\left(0^{-}\right)}, \phi \frac{1}{h} V_{\bar{z}}^{\left(0^{-}\right)}\right)^{I}(z, \bar{z})^{I}+E^{\left(1^{-}\right)}
$$

Here, $I$ ranges over ordered multi-indices of degree 2. We iterate this process and define:

$$
J_{N}=\sum_{j=0}^{N-1} \sum_{|I|=j} \psi\left(\phi \frac{1}{h} V_{z}^{\left(0^{-}\right)} z, \phi \frac{1}{h} V_{\bar{z}}^{\left(0^{-}\right)} \bar{z}\right)^{I} J_{1}
$$

so that

$$
J_{N} \mathcal{A}=\psi+\psi \sum_{|I|=N}\left(\phi \frac{1}{h} V_{z}^{\left(0^{-}\right)}, \phi \frac{1}{h} V_{\bar{z}}^{\left(0^{-}\right)}\right)^{I}(z, \bar{z})^{I}+E^{\left(1^{-}\right)}
$$

Finally, if we define:

$$
\widetilde{J}=J_{N}-\psi \sum_{|I|=N}\left(\phi \frac{1}{h} V_{z}^{\left(0^{-}\right)}, \phi \frac{1}{h} V_{\bar{z}}^{\left(0^{-}\right)}\right)^{I} f_{I} S^{(2)}
$$

(see Proposition 3.28 for the definition of $f_{I}$ and $S^{(2)}$; in the subscript of $f$, we are identifying $I$ with the underlying unordered multi-index) we see that:

$$
\widetilde{J} \mathcal{A}=\psi+E^{\left(1^{-}\right)}+\sum_{|I|=N} E^{\left(0^{-}\right)} f_{I} E^{\left(1^{-}\right)}
$$

Thus, we have constructed our parametrix, provided we do not mind multiplying by $C^{L}$ functions (here, given $L, f_{I}$ will be $C^{L}$ provided $N$ is large enough, see Proposition 3.28). By picking $L$ large enough for whichever regularity property we wish to show, Propositions 3.22 and 3.25 (the $L^{p}$ and $N L^{p}$ regularity) follow just as before. Unfortunately, the Lipschitz regularity does not follow immediately, since we have used operators that are smoothing of order $0^{-}$and not of order 0 .

Lemma 3.30. For every ordered multi-index I,

$$
\psi\left(\phi \frac{1}{h} V_{z}^{\left(0^{-}\right)} z, \phi \frac{1}{h} V_{\bar{z}}^{\left(0^{-}\right)} \bar{z}\right)^{I} \phi \mathcal{M} \pi^{(L)} \equiv \begin{cases}V^{(0)} D_{t}^{\frac{a+b}{2}-1} \pi^{(L)} & \text { if } a+b \text { is even } \\ V^{(1)} D_{t}^{\frac{a+b-1}{2}} \pi^{(L)} & \text { if } a+b \text { is odd }\end{cases}
$$

where $V^{(0)}: \Lambda_{\alpha} \rightarrow \Lambda_{\alpha}$, for all $\alpha>0$, and $V^{(1)}: \Lambda_{\alpha} \rightarrow \Lambda_{\alpha+\frac{1}{2}}$ for all $\alpha>0$. 
Proof. When $|I|=0$, we have the lemma by Equation (3.8). Thus, if $V_{z \text { and } \bar{z}}^{\left(0^{-}\right)}$were continuous $\Lambda_{\alpha, \mathrm{cpt}} \rightarrow \Lambda_{\alpha \text {, loc }}$, we would have the result-though, this is not quite the case. Consider, for ease of notation, a case when $|I|=2$ (the more general case follows in exactly the same way, and we leave such details to the reader)

$$
\psi \frac{1}{h} V_{z}^{\left(0^{-}\right)} z \phi \frac{1}{h} V_{\bar{z}}^{\left(0^{-}\right)} \bar{z} \phi \mathcal{M} \pi^{(L)}=\psi f_{0} R^{(0)} D_{t} L^{(2)} f_{1} \phi R^{(0)} D_{t} L^{(2)} f_{2} \phi \mathcal{M} \pi^{(L)}
$$

where on the RHS, we really mean a sum of such terms, and $f_{0}, f_{1}, f_{2} \in C^{\infty}$ may vary from term to term (see Proposition 3.29). Consider, if $\phi \prec \phi^{\prime}$,

$$
\begin{aligned}
\psi f_{0} R^{(0)} D_{t} L^{(2)} f_{1} \phi R^{(0)} D_{t} L^{(2)} f_{2} \phi \mathcal{M} \pi^{(L)} \\
=\psi f_{0} f_{1} R^{(0)} D_{t} L^{(2)} \phi R^{(0)} D_{t} L^{(2)} f_{2} \phi \mathcal{M} \pi^{(L)} \\
\quad+\psi f_{0}\left[R^{(0)} D_{t} L^{(2)}, f_{1} \phi^{\prime}\right] \phi R^{(0)} D_{t} L^{(2)} f_{2} \phi \mathcal{M} \pi^{(L)}
\end{aligned}
$$

Now, $\phi\left[R^{(0)} D_{t} L^{(2)}, f_{1} \phi^{\prime}\right]$ takes $\Lambda_{\alpha} \rightarrow \Lambda_{\alpha+\frac{1}{2}-\epsilon}$ for all $\epsilon>0$, since both

$$
R^{(0)} \phi^{\prime}\left[L^{(0)}, \phi^{\prime} f_{1}\right] \text { and }\left[R^{(0)}, \phi^{\prime} f_{1}\right] \phi^{\prime} L^{(0)}
$$

do by Proposition 2.11 (for the later equation, we use the analog of Proposition 2.11 with right convolution operators in place of left convolution operators everywhere). Thus, the second term on the RHS of the above equation is of the desired form, since $\mathcal{M} \pi^{(L)}$ satisfies the conclusion of the lemma, the commutator takes $\Lambda_{\alpha} \rightarrow \Lambda_{\alpha+\frac{1}{2}-\epsilon}$, and all other terms take $\Lambda_{\alpha} \rightarrow \Lambda_{\alpha-\epsilon}$ (for all $\alpha, \epsilon, \alpha-\epsilon>0$ ). In this manner, we see that we may commute $f_{2}$ to the front as well, leaving only error terms satisfy the conclusion of the lemma. Hence, we are left with considering

$$
\begin{aligned}
& \psi f_{0} f_{1} f_{2} R^{(0)} D_{t} L^{(2)} \phi R^{(0)} D_{t} L^{(2)} \phi \mathcal{M} \pi^{(L)} \\
& \quad=\psi f_{0} f_{1} f_{2} R^{(0)} D_{t} L^{(2)} \phi R^{(0)} D_{t} L^{(2)} \phi \begin{cases}D_{t}^{\frac{a+b}{2}-1}\left(D_{t} R^{(2)} \pi^{(L)}\right) & \text { if } a+b \text { is even, } \\
D_{t}^{\frac{a+b-1}{2}} R^{(1)} \pi^{(L)} & \text { if } a+b \text { is odd }\end{cases}
\end{aligned}
$$

We now use Remark 2.14 to see that the RHS of the above equation

$$
\equiv \psi f_{0} f_{1} f_{2} \begin{cases}\left(D_{t} R^{(2)}\right)\left(D_{t} L^{(2)}\right) D_{t}^{\frac{a+b}{2}-1} \pi^{(L)} & \text { if } a+b \text { is even } \\ R^{(1)}\left(D_{t} L^{(2)}\right) D_{t}^{\frac{a+b-1}{2}} \pi^{(L)} & \text { if } a+b \text { is odd }\end{cases}
$$

The regularity properties of the above operators completes the proof of the lemma (see Section 2.4).

The parametrix $\widetilde{J}$ consists of terms that satisfy the hypotheses of Lemma 3.30, along with terms that are smoothing of order $2^{-}$(where some of the terms that are smoothing of order $2^{-}$involve multiplication by a $C^{L}$ function, with $L$ as large as we choose, and so are not strictly smoothing of order $2^{-}$, but satisfy the proper regularity properties, provided $L$ is large enough depending on which regularity property we want). Thus, we have the following Lipschitz regularity:

Proposition 3.31. $\mathcal{A}$ loses at most $\frac{a+b}{2}-1$ derivatives near 0 in Lipschitz spaces; that is, if $\phi \prec \phi^{\prime} \prec \phi^{\prime \prime}$ are cut off functions supported sufficiently close to $0 \in \mathbb{H}^{1}$, and $u$ is a distribution, we have (for every $\alpha>0$ with $\alpha+\frac{a+b}{2}-1>0$ ),

$$
\|\phi u\|_{\Lambda_{\alpha}} \lesssim\left\|\phi^{\prime \prime} \mathcal{A} u\right\|_{\Lambda_{\alpha+\frac{a+b}{2}-1}}+\left\|\phi^{\prime \prime} u\right\|_{-\infty}
$$


In fact, if $a+b$ is even, for every $\epsilon>0$,

$$
\|\phi u\|_{\Lambda_{\alpha}} \lesssim\left\|\phi^{\prime} D_{t}^{\frac{a+b}{2}-1} \pi^{(L)} \phi^{\prime \prime} \mathcal{A} u\right\|_{\Lambda_{\alpha}}+\left\|\phi^{\prime \prime} \mathcal{A} u\right\|_{\Lambda_{\alpha-1+\epsilon}}+\left\|\phi^{\prime \prime} u\right\|_{-\infty}
$$

and if $a+b$ is odd, for every $\epsilon>0$,

$$
\|\phi u\|_{\Lambda_{\alpha}} \lesssim\left\|\phi^{\prime} D_{t}^{\frac{a+b-1}{2}} \pi^{(L)} \phi^{\prime \prime} \mathcal{A} u\right\|_{\Lambda_{\alpha-\frac{1}{2}}}+\left\|\phi^{\prime \prime} \mathcal{A} u\right\|_{\Lambda_{\alpha-1+\epsilon}}+\left\|\phi^{\prime \prime} u\right\|_{-\infty}
$$

Remark 3.32. It is possible that the $\epsilon$ in the above Proposition is an artifact of the proof. Perhaps a closer inspection of the operators involved would remove the $\epsilon$. We will not pursue this here.

\section{Optimality}

We next show that, if we restrict our attention to the case

$$
A_{3}=Z_{L} \sum_{c+d=a+b} z^{c} \bar{z}^{d} g_{c, d}^{(1)}(z, t)
$$

the regularity results in the previous section are optimal; ie, that $\mathcal{A}$ loses precisely $\frac{a+b}{2}-1$ derivatives in $L^{p}$ Sobolev spaces and Lipschitz spaces near 0 . Indeed, we will show:

Proposition 4.1. Given $\alpha>0$, suppose that there is $a \beta<\alpha$ and $0<\alpha_{0}<\alpha$ and that there exist $\phi, \phi^{\prime} \in C_{0}^{\infty}$ which are equal to 1 on a neighborhood of $0 \in \mathbb{H}^{1}$, such that for every function $u \in C^{\infty}$, we have:

$$
\|\phi u\|_{\Lambda_{\alpha}} \lesssim\left\|\phi^{\prime} \mathcal{A} u\right\|_{\Lambda_{\alpha-\beta}}+\left\|\phi^{\prime} u\right\|_{\Lambda_{\alpha_{0}}}
$$

then, $\beta \leq 1-\frac{a+b}{2}$.

and

Proposition 4.2. Given $s \in \mathbb{R}$ and $1<p<\infty$, suppose that there is an $r \in \mathbb{R}$ and $a s_{0}<s$ and that there exist $\phi, \phi^{\prime} \in C_{0}^{\infty}$ which are equal to 1 on a neighborhood of $0 \in \mathbb{H}^{1}$, such that for every function $u \in C^{\infty}$, we have:

$$
\|\phi u\|_{L_{s}^{p}} \lesssim\left\|\phi^{\prime} \mathcal{A} u\right\|_{L_{s-r}^{p}}+\left\|\phi^{\prime} u\right\|_{L_{s_{0}}^{p}}
$$

then, $r \leq 1-\frac{a+b}{2}$.

We will be using the proof methods from [BDKT06, Koh05]. We define

$$
v(z, t)=\exp \left(-\left(|z|^{2}-i t\right)\right)
$$

and for $0<\lambda \in \mathbb{R}$,

$$
v_{\lambda}(z, t)=v\left(\lambda z, \lambda^{2} t\right)=v\left(\gamma_{\lambda}(z, t)\right)
$$

Let $\psi \in C_{0}^{\infty}$ be an arbitrary function that equals 1 on a neighborhood of $0 \in \mathbb{H}^{1}$. We will prove (for each $\delta>0, s^{\prime} \in \mathbb{R}$, and for $\lambda \gg 1$ ):

$$
\begin{gathered}
\left\|\psi v_{\lambda}\right\|_{\Lambda_{\delta}} \approx \lambda^{2 \delta} \\
\left\|\psi \mathcal{A} v_{\lambda}\right\|_{\Lambda_{\delta}} \lesssim \lambda^{2-a-b+2 \delta} \\
\left\|\psi v_{\lambda}\right\|_{L_{s^{\prime}}^{p}} \approx \lambda^{2 s^{\prime}-2 / p} \\
\left\|\psi \mathcal{A} v_{\lambda}\right\|_{L_{s^{\prime}}^{p}} \lesssim \lambda^{2-a-b+2 s^{\prime}-2 / p}
\end{gathered}
$$


Here, the implicit constants depend on $p, s^{\prime}, \delta$, and $\psi$. Let's first see how these results will yield Propositions 4.1 and 4.2. Indeed, suppose we satisfy the hypotheses of Proposition 4.1, and so we have the inequality:

$$
\|\phi u\|_{\Lambda_{\alpha}} \lesssim\left\|\phi^{\prime} \mathcal{A} u\right\|_{\Lambda_{\alpha-\beta}}+\left\|\phi^{\prime} u\right\|_{\Lambda_{\alpha_{0}}}
$$

Thus, for $\lambda \gg 1$,

$$
\begin{aligned}
\lambda^{2 \alpha} & \lesssim\|\phi u\|_{\Lambda_{\alpha}} \\
& \lesssim\left\|\phi^{\prime} \mathcal{A} u\right\|_{\Lambda_{\alpha-\beta}}+\left\|\phi^{\prime} u\right\|_{\Lambda_{\alpha_{0}}} \\
& \lesssim \lambda^{2-a-b+2(\alpha-\beta)}+\lambda^{2 \alpha_{0}}
\end{aligned}
$$

Using that $\lambda^{2 \alpha} \not \lambda^{2 \alpha_{0}}$, we must have:

$$
\lambda^{2 \alpha} \lesssim \lambda^{2-a-b+2(\alpha-\beta)}
$$

It follows that $2 \alpha \leq 2-a-b+2(\alpha-\beta)$. That is, $2 \beta \leq 2-a-b$, which would complete the proof of Proposition 4.1. The same proof with the equations (4.3) and (4.4) in place of (4.1) and (4.2) yields Proposition 4.2. We only outline the proofs of these equations as they are elementary.

The first step is to write $\mathcal{A} v_{\lambda}$ in a form that is easier to understand. Indeed, we will write $w(z, t)$ for any function of the form:

$$
z^{d} \bar{z}^{e} v(z, t)
$$

where $d, e \in \mathbb{N}$. We will also write $w_{\lambda}(z, t)$ for a function of the form $w\left(\lambda z, \lambda^{2} t\right)$.

Lemma 4.3. $\mathcal{A} v_{\lambda}$ is a finite linear combination of terms of the form:

$$
\lambda^{\delta} g(z, t) w_{\lambda}(z, t)
$$

where $g \in C^{\infty}$ and $\delta \leq 2-a-b$. Here, $g$ is independent of $\lambda$ (but may vary from term to term).

Proof. We leave the proof to the reader.

Remark 4.4. Lemma 4.3 is the only place where we use the assumption

$$
A_{3}=Z_{L} \sum_{c+d=a+b} z^{c} \bar{z}^{d} g_{c, d}^{(1)}(z, t)
$$

In fact, Lemma 4.3 works more naturally on the class of operators given by $\mathcal{A}^{*}$. We now see the trade off: it is easier to prove regularity for $\mathcal{A}$ and lack of regularity for $\mathcal{A}^{*}$, as one would expect.

Let $\psi \in C_{0}^{\infty}$ be an arbitrary function that equals 1 on a neighborhood of $0 \in \mathbb{H}^{1}$. The following inequalities are easy to prove, and complete the proofs of Propositions 4.1 and 4.2. For each $\delta>0, s^{\prime} \in \mathbb{R}$, and for $\lambda \gg 1$,

$$
\begin{gathered}
\left\|\psi v_{\lambda}\right\|_{\Lambda_{\delta}} \approx \lambda^{2 \delta} \\
\left\|\psi w_{\lambda}\right\|_{\Lambda_{\delta}} \lesssim \lambda^{2-a-b+2 \delta} \\
\left\|\psi w_{\lambda}\right\|_{L_{s^{\prime}}^{p}} \approx \lambda^{2 s^{\prime}-2 / p}
\end{gathered}
$$

Here, the implicit constants depend on $\psi, \delta, s^{\prime}$, and the particular $d$ and $e$ in the definition of $w_{\lambda}$. 
Remark 4.5. In the proof of optimality, we do not use that $h(0) \neq 0$. Thus, we see that if $\mathcal{A}=Z_{L} \bar{Z}_{L}+\bar{Z}_{L} h(\zeta) Z_{L}$, and $h$ vanishes to infinite order in the $z$ variable as $\zeta \rightarrow 0$ (that is, for every $\alpha$ a multi-index, $h$ is of the form $(z, \bar{z})^{\alpha} g_{\alpha}$, where $\left.g_{\alpha} \in C^{\infty}\right)$, then $\mathcal{A}$ is not hypoelliptic near 0 .

\section{Some Closing Remarks}

By conjugating the operator $\mathcal{A}$ by translation on the left, one can replace it with an operator that vanishes at $z=z_{0}$, instead of $z=0$. For instance, consider the following example:

Example 5.1. Let $\mathcal{A}$ be given by

$$
Z_{L} \bar{Z}_{L}+\bar{Z}_{L}|z|^{2 k}|z-1|^{2 j} Z_{L}
$$

From our parametrix construction we know that $\mathcal{A}$ loses $k-1$ derivatives at $z=0$. If we conjugate $\mathcal{A}$ by translation on the left by $(1,0)$ (using the Heiseneberg group structure) $\mathcal{A}$ becomes:

$$
Z_{L} \bar{Z}_{L}+\bar{Z}_{L}|z+1|^{2 k}|z|^{2 j} Z_{L}
$$

Thus, the untranslated $\mathcal{A}$ loses $j-1$ derivatives at $z=1$. Finally, $\mathcal{A}$ is subelliptic everywhere else by a proof along the lines of Remark 3.5.

The construction of the parametrix should be considered as taking place in two parts. The first part is Theorem 3.3, while the second is the computation of the inverse of the Toeplitz operator $\pi^{(L)} A_{2}^{\prime} \pi^{(L)}$; ie, the computation of $\mathcal{M}$. All other considerations, we believe, are secondary.

The proof of Theorem 3.3 relied on a procedure of "freezing coefficients." That is, we studied the operator

$$
\mathcal{B}_{\alpha}=Z_{L} \bar{Z}_{L}+\alpha \bar{Z}_{L} Z_{L}
$$

$\mathcal{B}_{\alpha}$ had a fundamental solution $T_{\alpha}$ which was meromorphic in $\alpha$, with a simple pole at $\alpha=0$. Or, if we set $S_{\alpha}=\alpha T_{\alpha}, \mathcal{B}_{\alpha} S_{\alpha}=\alpha$, and $S_{\alpha}$ depends smoothly on $\alpha$. We note that all we really needed in the proof was:

$$
\mathcal{B}_{\alpha} S_{\alpha}=\alpha+E^{(1)}
$$

where $E^{(1)}$ is smoothing of order 1 (we mean this equation locally, ie on a sufficiently small neighborhood of 0). Using the results of [FS74], we may replace (in Equation (5.1)) $\bar{Z}_{L}$ with $\overline{\partial_{b}}$ on any strictly pseudoconvex CR manifold of dimension 3 . That is, we approximate $\overline{\partial_{b}}$ by $\bar{Z}_{L}$ (and the adjoint of $-\overline{\partial_{b}}$ by $Z_{L}$ ) and then Equation (5.1) follows by approximating by the result on $\mathbb{H}^{1}$.

Moreover, we could have replaced $X_{L}$ and $Y_{L}$ in Equation (5.1) by any two vector fields $(X$ and $Y)$ such that $X, Y$, and $[X, Y]$ span the tangent space at every point (and replace $\bar{Z}_{L}$ by $\frac{1}{2}(X+i Y)$ and $-Z_{L}$ by its adjoint) and then used the results of [RS76] to "lift" these operators and then approximate them by the Heisenberg group (note that $X$ and $Y$ could be on a manifold of dimension less than 3). Thus, we get Equation (5.1) even in this case.

If one were to want to study a higher dimensional analog of Equation (5.1), one could replace the 3 dimensional Heisenberg group with its $2 n+1$ dimensional analog, $\mathbb{H}^{n}$ (see [Ste93]). The Lie algebra of $\mathbb{H}^{n}$ is spanned by:

$$
X_{j}=\partial_{x_{j}}+2 y_{j} \partial_{t}, \quad Y_{j}=\partial_{y_{j}}-2 x_{j} \partial_{t}, \quad\left[X_{j}, Y_{j}\right]=-4 \partial_{t}
$$


We set $\bar{Z}_{j}=\frac{1}{2}\left(X_{j}+i Y_{j}\right)$ and $Z_{j}=\frac{1}{2}\left(X_{j}-i Y_{j}\right)$. Finally, for $\alpha=\left(\alpha_{1}, \ldots, \alpha_{n}\right)$, we set:

$$
\mathcal{B}_{\alpha}=\sum_{j=1}^{n}\left(Z_{j} \bar{Z}_{j}+\alpha_{j} \bar{Z}_{j} Z_{j}\right)
$$

Then, $\mathcal{B}_{\alpha}$ has a fundamental solution $T_{\alpha}$ (for $\alpha$ near 0 but not equal to 0 ), and

$$
\left(\sum_{j=1}^{n} \alpha_{j}\right) T_{\alpha}
$$

is holomorphic in $\alpha$ for $\alpha$ near 0 .

In fact, Heisenberg algebras are the only nilpotent Lie algebras where this sort of phenomenon appears (see [HN05], Theorem 8.9 on page 86). Therefore, if one wishes to generalize our parametrix construction to vector fields that take more than 2 commutators to span the tangent space, one may not approximate by a nilpotent Lie algebra. In some such "higher step" cases, one can prove an analog of Equation (5.1), using NIS operators. We say no more about this, here.

Define $\bar{L}=\partial_{x}+i x \partial_{y}$ and $L=\partial_{x}-i x \partial_{y}$ on $\mathbb{R}^{2}$. It is shown in [Str06] that the method of parametrix construction in this paper applies to the operator:

$$
\mathcal{A}=L \bar{L}+\bar{L} x^{2 k} L
$$

$(k \in \mathbb{N}, k>0)$ It is shown in [Chr05], however, that $\mathcal{A}+\partial_{s}^{2}$ (as an operator on $\mathbb{R}^{3}$ ) is not hypoelliptic. One of the main reasons our parametrix construction fails for $\mathcal{A}+\partial_{s}^{2}$ is that when we "freeze coefficients," we are left with the operator:

$$
L \bar{L}+\alpha \bar{L} L+\partial_{s}^{2}
$$

which is not hypoelliptic for $\alpha<0$. One might note that (in this case) we only use $S_{\alpha}$ for $\alpha>0$ not $\alpha<0$, but the only way we know to prove good estimates on the kernel of $S_{\alpha}$ is to use its values for $\alpha$ on a circle around 0 along with Cauchy's formula (see [Str06]).

The only other parametrix construction that we know of for operators like Kohn's, is that of Parenti and Parmeggiani [PP06]. Their parametrix construction and ours might seem quite different but, in fact, are very related. The operators they study are defined in terms of the vector fields $L_{j}(j=1, \ldots, n)$ on $\mathbb{R}^{n+1}$, where

$$
L_{j}=\partial_{x_{j}}-i \mu_{j} x_{j} \partial_{y}
$$

where the coordinates of $\mathbb{R}^{n+1}$ are given by $x_{1}, \ldots, x_{n}, y$. Here, $\mu_{1}, \ldots, \mu_{n}$ are positive real numbers that are rationally independent. Fix $d \in \mathbb{N}$ and for each multi-index $\alpha$ with $|\alpha|=d$, pick a positive real number $c_{\alpha}$ (at least one of which is nonzero). Define

$$
Q(x)=\sum_{|\alpha|=d} c_{\alpha} x^{2 \alpha}
$$

Let $\gamma \in \mathbb{R}$ be a real number and consider the operator

$$
\mathcal{A}=\sum_{j=1}^{n}\left(L_{j}^{*} L_{j}+L_{j} Q(x) L_{j}^{*}\right)+i\left(\gamma+\sum_{j=1}^{n} \mu_{j}\right) \partial_{y}
$$


Suppose $\pi$ is the projection onto the $L^{2}$ kernel of

$$
\sum_{j=1}^{n} L_{j}^{*} L_{j}+i\left(\gamma+\sum_{j=1}^{n} \mu_{j}\right) \partial_{y}
$$

Then, the operator $\Lambda_{1-d}\left(D_{y}\right)$ in [PP06] is precisely the operator such that:

$$
\pi \mathcal{A} \pi=\Lambda_{1-d}\left(D_{y}\right) \pi
$$

Hence, by inverting $\Lambda_{1-d}$, they are inverting the Toeplitz operator $\pi \mathcal{A} \pi$. This gives us insight into why they chose to have the $\mu_{j}$ s be rationally independent. Indeed, when the $\mu_{j}$ s are rationally independent, after taking the Fourier transform $y \rightarrow \eta$, and fixing $\eta, \pi^{(\eta)}$ becomes projection onto a one dimensional space, thereby making the Toeplitz operator $\pi \mathcal{A} \pi$ (and its inverse) easy to compute. If the $\mu_{j}$ were rationally dependent, $\pi$ might be a projection onto a higher dimensional space, complicating matters. This shows us one way in which Kohn's operator is more complicated than the above operator. Indeed, the Toepliz operator corresponding to Kohn's operator $\left(\pi^{(L)} A_{2}^{\prime} \pi^{(L)}\right)$ is much more difficult to reduce to a one dimensional problem, or even a finite dimensional problem. This is why the parametrix construction in [Str06] is much simpler than the parametrix construction in this paper. It seems likely that the methods here (or in [Str06]) could be used to prove $L^{p}$ Sobolev and Lipschitz regularity for the operators discussed in [PP06].

In fact, one can formally follow the parametrix construction of [PP06], and replace the pseudodifferential operators of [BdM74] with the pseudodifferential operators of [NS79] and obtain another construction for the parametrix discussed in [Str06]. This is not immediate, and requires a bit of work. The construction turns out to be much more complicated than that in [Str06], and it seems difficult (if not impossible) to extend the construction to prove $L^{p}$ regularity for Kohn's operator. We, therefore, do not discuss this line of reasoning any further.

We see, from the remarks above, that one of the main aspects of our parametrix construction was the inversion of specific Toepliz operators. It is worth remarking that the calculus of [BdMG81] does not seem to help us in inverting these Toepliz operators. Indeed, it seems impossible to reduce our Toepliz operators to any sort of "elliptic Toeplitz operators," in the sense of [BdMG81].

Our inversion of $\pi^{(L)} A_{2}^{\prime} \pi^{(L)}$ on $\pi^{(L)}$ seems very tied to the group structure on the Heisenberg group. In the case of Kohn's operator (that is, in the case $a=b$ ), one can invert $\pi^{(L)} A_{2}^{\prime} \pi^{(L)}$ in another way by diagonalizing it. Indeed, one may diagonalize $\pi^{(L)} A_{2}^{\prime} \pi^{(L)}$ by taking the Fourier transform $t \rightarrow \tau$, writing $z=r e^{i \theta}$, and taking the Fourier transform $\theta \rightarrow n$. Then, inverting it (at least formally) is easy. Proving the deeper regularity properties, in this manner, is a bit more difficult than the methods outlined in this paper and does not give much hope for a far reaching generalization, though might cover other cases. This procedure was the one alluded to in [Str06].

\section{REFERENCES}

[BDKT06] A. Bove, M. Derridj, J.J. Kohn, and D.S. Tartakoff, Hypoellipticity for a sum of squares of complex vector fields with large loss of derivatives, Math. Res. Lett. 13 (2006), no. 5, 683-702.

[BdM74] L. Boutet de Monvel, Hypoelliptic operators with double characteristics and related pseudo-differential operators, Comm. Pure Appl. Math. 27 (1974), 585-639. 
[BdMG81] L. Boutet de Monvel and V. Guillemin, The spectral theory of Toeplitz operators, Annals of Mathematics Studies, vol. 99, Princeton University Press, Princeton, NJ, 1981.

[CG84] M. Christ and D. Geller, Singular integral characterizations of Hardy spaces on homogeneous groups, Duke Math. J. 51 (1984), no. 3, 547-598.

[CGGP92] M. Christ, D. Geller, P. Głowacki, and L. Polin, Pseudodifferential operators on groups with dilations, Duke Math. J. 68 (1992), no. 1, 31-65.

[Chr05] M. Christ, A remark on sums of squares of complex vector fields, 2005, arXiv:math.CV/0503506.

[Fol75] G.B. Folland, Subelliptic estimates and function spaces on nilpotent Lie groups, Ark. Mat. 13 (1975), no. 2, 161-207.

[FS74] G.B. Folland and E.M. Stein, Estimates for the $\bar{\partial}_{b}$ complex and analysis on the Heisenberg group, Comm. Pure Appl. Math. 27 (1974), 429-522.

[Hel86] P. Heller, Analyticity and regularity for nonhomogeneous operators on the heisenberg group, Princeton University Dissertation (1986).

[HN05] B. Helffer and F. Nier, Hypoelliptic estimates and spectral theory for Fokker-Planck operators and Witten Laplacians, Lecture Notes in Mathematics, vol. 1862, SpringerVerlag, Berlin, 2005.

[Hör67] L. Hörmander, Hypoelliptic second order differential equations, Acta Math. 119 (1967), 147-171.

[JT06] J-L. Journé and J-M. Trépreau, Hypoellipticité sans sous-ellipticité: le cas des systèmes de $n$ champs de vecteurs complexes en $(n+1)$ variables, Seminaire: Equations aux Dérivées Partielles. 2005-2006, Sémin. Équ. Dériv. Partielles, École Polytech., 2006, pp. Exp. No. XIV, 19.

[Koe02] K.D. Koenig, On maximal Sobolev and Hölder estimates for the tangential CauchyRiemann operator and boundary Laplacian, Amer. J. Math. 124 (2002), no. 1, 129197.

[Koh05] J.J. Kohn, Hypoellipticity and loss of derivatives, with an appendix by M. Derridj and D. Tartakoff, Ann. of Math. (2) 162 (2005), no. 2, 943-986.

[NS79] A. Nagel and E.M. Stein, Lectures on pseudodifferential operators: regularity theorems and applications to nonelliptic problems, Mathematical Notes, vol. 24, Princeton University Press, Princeton, N.J., 1979.

[PP05] C. Parenti and A. Parmeggiani, On the hypoellipticity with a big loss of derivatives, Kyushu J. Math. 59 (2005), no. 1, 155-230.

[PP06] , A note on Kohn's and Christ's examples, Hyperbolic problems and regularity questions, Trends Math., Birkhäuser, Basel, 2006, pp. 151-158.

[RS76] L.P. Rothschild and E.M. Stein, Hypoelliptic differential operators and nilpotent groups, Acta Math. 137 (1976), no. 3-4, 247-320.

[Ste70] E.M. Stein, Singular integrals and differentiability properties of functions, Princeton Mathematical Series, No. 30, Princeton University Press, Princeton, N.J., 1970.

[Ste82] An example on the Heisenberg group related to the Lewy operator, Invent. Math. 69 (1982), no. 2, 209-216.

[Ste93] Harmonic analysis: real-variable methods, orthogonality, and oscillatory integrals, Princeton Mathematical Series, vol. 43, Princeton University Press, Princeton, NJ, 1993.

[Str06] B. Street, $L^{p}$ regularity for Kohn's operator, Math. Res. Lett. 13 (2006), no. 5, 703711. 